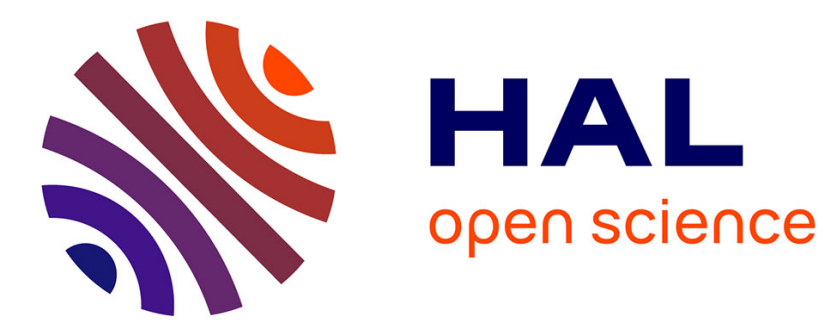

\title{
Group-nets et réseaux lexicographiques. Expressivité et décidabilité
}

Goulven Guillou

\section{To cite this version:}

Goulven Guillou. Group-nets et réseaux lexicographiques. Expressivité et décidabilité. Revue des Sciences et Technologies de l'Information - Série TSI: Technique et Science Informatiques, 2009, Numéro spécial "Réseaux de Petri", 28, pp.1171 - 1201. hal-01102905

\section{HAL Id: hal-01102905 \\ https://hal.univ-brest.fr/hal-01102905}

Submitted on 13 Jan 2015

HAL is a multi-disciplinary open access archive for the deposit and dissemination of scientific research documents, whether they are published or not. The documents may come from teaching and research institutions in France or abroad, or from public or private research centers.
L'archive ouverte pluridisciplinaire HAL, est destinée au dépôt et à la diffusion de documents scientifiques de niveau recherche, publiés ou non, émanant des établissements d'enseignement et de recherche français ou étrangers, des laboratoires publics ou privés. 


\title{
Group-nets et réseaux lexicographiques : expressivité et décidabilité.
}

\author{
Goulven GUILLOU
}

\author{
LISyC-EA 3883 \\ Département d'informatique \\ U.F.R. des sciences et techniques \\ Université de Bretagne Occidentale \\ 6, av. Le Gorgeu 29200 Brest, FRANCE \\ goulven.guillou@univ-brest.fr
}

\begin{abstract}
RÉSUMÉ. Les motivations liées aux extensions algébriques des réseaux de Petri portent entre autres sur des problèmes de modélisation, de vision unifiée ou abstraite des grandes classes de réseaux de Petri et d'obtention de modèles plus expressifs limitant les pertes du point de vue de la décidabilité. Nous proposons deux grandes classes de réseaux, les group - nets et les réseaux lexicographiques, la première se déclinant elle-même en plusieurs sous-classes. Nous étudions les liens entre ces réseaux et différentes classes usuelles de réseaux de Petri en donnant, dans le cas d'inclusions, les algorithmes permettant de passer de l'un à l'autre. Des outils sont donnés pour résoudre certains problèmes de décidabilité pour les strict-ZZZ-nets et les $\mathbb{Z}$-nets. On montre, en revanche, que les réseaux lexicographiques ont la puissance des machines de Turing rendant tout problème non trivial les concernant indécidable.

ABSTRACT. Algebraically generalized Petri nets are motivated by, among others, problems of modelling, the need to obtain an abstract and general frame of the most part of the common classes of Petri nets and the wish to obtain more expressive models which restrict loss at the level of the decidability. We propose two classes of nets, group - nets and lexicographic nets, the former containing itself several subclasses. We study the links between these nets and various classes of usual Petri nets. In the case of inclusions, we exhibit the algorithms which allow to pass from a class to the other one. Tools are given to resolve some decidability problems for strict-ZZZ-nets and $\mathbb{Z}$-nets. We show that lexicographic nets are Turing powerful, therefore all non-trivial problems are undecidable on this class.
\end{abstract}

MOTS-CLÉS : extensions algébriques des réseaux de Petri, expressivité, décidabilité KEYWORDS: algebraically generalised Petri nets, expressiveness, decidability

RSTI - TSI - 28/2009. Réseaux de Petri et algorithmes, pages 1171 à 1201 


\section{Introduction}

Le succès des réseaux de Petri dans le domaine des systèmes dynamiques concurrents à événements discrets provient du fait qu'ils offrent à la fois un outil graphique naturel et intuitif pour la modélisation de tels systèmes et un panel d'outils algébriques pour leur description et leur analyse. De nombreuses extensions des réseaux de Petri places/transitions usuels ont été proposées soit pour réduire la taille des modélisations, soit pour étendre leur pouvoir d'expression, soit pour disposer d'informations plus précises sur les jetons, soit encore, d'un point de vue théorique, pour essayer d'obtenir un modèle plus abstrait dégageant ainsi potentiellement de nouveaux outils sur les réseaux usuels. Certaines de ces extensions sont des réseaux de haut-niveau qui permettent de construire des représentations plus compactes mais n'augmentent pas le pouvoir d'expression des réseaux de Petri : ces réseaux de haut-niveau peuvent être modélisés par des réseaux de Petri équivalents et sont donc généralement appelés des abréviations de réseaux. Les réseaux colorés (Jensen, 1992), qui distinguent les jetons, les réseaux à capacité (André, 1981) ou les réseaux à prédicats (Genrich $e t$ al., 1979) en font partie. D'autres extensions modifient considérablement la sémantique du modèle standard. C'est le cas par exemple des réseaux de Petri continus, des réseaux de Petri hybrides (David et al., 1998) ou encore des réseaux synchronisés (Moalla et al., 1978). D'autres extensions encore sont obtenues par ajout d'arcs spécifiques (arc Reset (Araki et al., 1977), arc inhibiteur (Hack, 1975)...), par modification de la règle de tir (Self-Modifying nets (Valk, 1978), G-réseaux (Dufourd et al., 1998) ...), ou par remplacement de l'algèbre usuelle (le monoïde $(\mathbb{N},+, 0)$ des entiers naturels) par une algèbre plus générale pour donner des réseaux qualifiées d'extensions algébriques. Pour ce dernier cas, le travail de (Meseguer et al., 1990) présente une étude très générale de l'algèbre mise en oeuvre dans les réseaux de Petri. On peut mentionner les Abstract Petri Nets de (Padberg, 1996) qui tendent à fournir un modèle abstrait et uniforme des réseaux de Petri en général. Ces extensions constituent des extensions strictes, c'est-à-dire des réseaux qui ne sont pas modélisables par des réseaux de Petri standard. Une des grandes préoccupations liées aux extensions strictes des réseaux de Petri est d'essayer de préserver les propriétés de décidabilité des réseaux de Petri usuels. On sait par exemple que les problèmes de terminaison, de couverture, du caractère borné d'un réseau, du caractère borné d'une place, de l'accessibilité, du blocage et de la vivacité sont des problèmes ayant une solution effective dans la classe des réseaux de Petri. Dans ce contexte on peut citer les Process Rewrite Systems (Mayr, 2000) et les réseaux de Petri récursifs (Haddad et al., 2007) qui préservent la décidabilité de l'accessibilité ou encore les data nets (Lazić et al., 2007) pour lesquels la couverture et la terminaison restent décidables ainsi que le caractère borné du réseau sous certaines restrictions.

Le présent article propose des classes de réseaux de Petri obtenues en modifiant à chaque fois l'algèbre des places et en adaptant, en conséquence, la règle de tir. La principale motivation de ce travail consiste à obtenir une extension des réseaux de Petri tout en préservant la décidabilité des (ou de certains) problèmes évoqués cidessus. Dans ce cadre des algorithmes de décisions sont donnés pour certaines classes 
de ces réseaux. Par ailleurs, afin de connaître le pouvoir d'expression de nos modèles, nous les comparons à des modèles connus. Nous ébauchons ainsi une classification de nos réseaux par rapport à des réseaux classiques comme les réseaux de Petri bornés ou les réseaux de Petri à arcs inhibiteurs et donnons, dans certains cas d'inclusion, les algorithmes permettant de passer d'un type de réseaux à un autre.

L'article est organisé comme suit : après un bref rappel des définitions nécessaires à la lecture, la section 3 traite globalement des group-nets en commençant par les strict-group-nets. Ce qui motive l'introduction des strict-group-nets où l'algèbre des places est un groupe privé de son élément neutre est leur capacité à modéliser de façon extrêmement compacte des cycles dans des systèmes de transitions étiquetées via l'utilisation naturelle de groupes cycliques. Mais cette piste est rapidement délaissée car le lien de l'algèbre des réseaux de Petri avec le groupe $\mathbb{Z}$ incite vivement à étudier les strict-ZZ-nets. La possibilité de définir un ordre partiel bien fondé sur $\mathbb{Z}$ permet alors de s'attaquer aux problèmes de la décidabilité du caractère borné d'un réseau et plus particulièrement d'une place. Les strict-ZZ-nets n'étendant pas les réseaux de Petri excepté lorsqu'ils sont purs, nous proposons une extension commune appelée $\mathbb{Z}$-net en adjoignant un arc particulier dit «inconditionnel ». Les $\mathbb{Z}$-nets conservent des propriétés intéressantes des strict- $\mathbb{Z}$-nets et ne sont pas comparables en particulier aux réseaux de Petri à arcs inhibiteurs. Le fait que la notion de ressource stricto sensu disparaisse rend le fonctionnement de ces réseaux peu intuitif et les rend donc difficiles à manipuler. Mais le fait que l'on retrouve ou que l'on étende quelques grandes classes des réseaux de Petri (réseaux de Petri, réseaux de Petri purs, réseaux de Petri bornés, réseaux élémentaires) à partir des group-nets pour des groupes particuliers permet d'avoir une vision uniforme de ces classes et justifie l'intérêt de ce nouveau modèle. Pour se rapprocher encore plus des réseaux de Petri où une transition s'effectue en deux phases (une phase de consommation suivie d'une phase de production), nous définissons une classe de réseaux, les group $_{2}-$ nets, qui permet d'obtenir une vision unifiée des strict- $\mathbb{Z}$-nets et des $\mathbb{Z}$-nets. Dans la section 4 , nous présentons les réseaux lexicographiques qui sont issus d'une axiomatisation de la notion de ressource et de la règle de tir des réseaux de Petri usuels et sont censés, par conséquent, se comporter comme eux. Les réseaux lexicographiques forment également une extension algébrique des réseaux de Petri avec une notion de ressource très étrange : on peut consommer strictement indéfiniment! Ces nouveaux réseaux ont malheureusement la puissance des machines de Turing. Cependant, nous montrons que les réseaux lexicographiques bornés sont les réseaux de Petri bornés ce qui achève d'une certaine manière leur description. Enfin, la conclusion en section 5 propose un certain nombre de pistes de recherche sur les deux modèles étudiés.

\section{Préliminaires}

Commençons par fixer la terminologie utilisée dans ce document. Un réseau de Petri est un quadruplet $R=\left(P, T, f, M_{0}\right)$ où : $P$ est un ensemble fini de places, $T$ est un ensemble fini de transitions (disjoint de $P$ ), $f: P \times T \cup T \times P \longrightarrow \mathbb{N}$ est la rela- 
tion de flot et $M_{0} \in \mathbb{N}^{P}$ est le marquage initial. Un réseau de Petri sera dit pur s'il ne comporte pas de self-loop c'est-à-dire si $\forall p \in P, \forall t \in T, f(p, t) * f(t, p)=0$. On peut effectuer le tir d'une transition $t \in T$ à partir d'un marquage $M \in \mathbb{N}^{P}$, noté $M[t\rangle$, si pour toute place $p \in P$ on a $M(p) \geq f(p, t)$. Le tir d'une transition $t$ permet d'obtenir le nouveau marquage $M^{\prime}$ défini par $: \forall p \in P, M^{\prime}(p)=M(p)-f(p, t)+f(t, p)$. Ce tir est noté $M[t\rangle M^{\prime}$. On dira que le marquage $M^{\prime}$ est accessible à partir du marquage $M$. Une séquence de transitions $\sigma=t_{1} t_{2} \ldots t_{n}$ est franchissable à partir du marquage $M$ si il existe une suite de marquages $M_{1}, M_{2} \ldots M_{n}$ tels que $M\left[t_{1}\right\rangle M_{1}\left[t_{2}\right\rangle M_{2} \ldots M_{n-1}\left[t_{n}\right\rangle M_{n}$. Le franchissement de $\sigma$ sera noté $M[\sigma\rangle M_{n}$. Le graphe des marquages d'un réseau de Petri $R=\left(P, T, f, M_{0}\right)$ est un graphe dont les sommets sont étiquetés par les marquages accessibles à partir de $M_{0}$ et dont les arcs correspondent aux franchissements de transition faisant transiter d'un marquage à l'autre. Un réseau de Petri est dit borné s'il existe un majorant $n \in \mathbb{N}$ du nombre de marques figurant dans chaque place pour tout marquage accessible à partir du marquage initial, on dira aussi qu'il est $n$-borné si $n$ est connu. Le langage d'un réseau de Petri est l'ensemble des séquences de franchissements valides à partir du marquage initial. Le langage d'un réseau de Petri est donc nécessairement préfixe. Deux réseaux sont dits équivalents si leurs graphes des marquages sont isomorphes. Ce choix est dû au fait que, d'une part, nous nous interdisons l'étiquetage et que, d'autre part, cette notion est centrale dans la synthèse de réseau, outil que nous utiliserons à plusieurs reprises dans la suite. Le problème de synthèse consiste à construire un réseau, après avoir décidé de son existence, dont le graphe des marquages est isomorphe à un graphe donné. Trivialement, nous avons le résultat suivant.

\section{Lemme 2.1 L'équivalence des réseaux est plus fine que l'égalité des langages.}

En effet deux réseaux équivalents ont nécessairement le même langage (à isomorphisme près des alphabets), par contre, deux réseaux ayant même langage ne sont pas nécessairement équivalents. Il suffit pour s'en convaincre d'analyser la figure 1 qui présente deux réseaux dont le langage commun est l'ensemble des préfixes de $a^{*}$ ainsi que leurs graphes des marquages (dessinés à droite des réseaux).

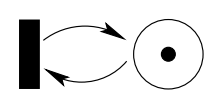

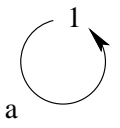
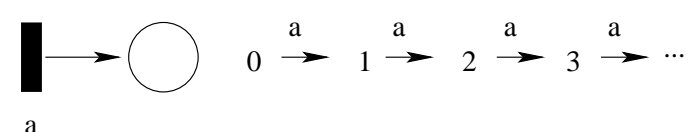

Figure 1. Deux RdP ayant même langage mais dont les graphes de marquages ne sont pas isomorphes

Nous utilisons par la suite cette équivalence pour comparer deux réseaux de classes différentes (i.e. pas nécessairement des réseaux de Petri) à partir du moment où une notion de graphe des marquages est définie. Nous renvoyons le lecteur à l'article de (Murata, 1989) pour une définition précise de l'arbre et du graphe de couverture d'un 
réseau de Petri. Rappelons simplement qu'il s'agit de structures finies qui donnent une description partielle de l'ensemble des marquages accessibles du réseau et qui permettent de vérifier de nombreuses propriétés comme la terminaison, la couverture ou le caractère borné d'une place ou d'un réseau. Bien qu'il ne s'agisse pas d'une présentation habituelle, les réseaux élémentaires ( $E N$ pour Elementary Nets) peuvent être vus comme des réseaux purs et 1-bornés (sous couvert que les arcs ne peuvent être pondérés que par 0 ou 1 mais dans le cadre de l'équivalence choisie cette contrainte importe peu).

Terminons cette section par quelques rappels mathématiques. Un groupe est une structure $(G,+, 0)$ où $G$ est un ensemble, + est une loi interne, associative, d'élément neutre 0 , telle que tout élément $x$ de $G$ admet un inverse $y$ (i.e. $x+y=y+x=0$ ). Si la loi est commutative, le groupe est dit commutatif ou abélien. Soit $x$ un élément de $G$, on appelle ordre de $x$, s'il existe, le plus petit entier positif non nul tel que $x+x+x+\ldots+x=0$ où $x$ apparaît $n$ fois. Si un tel entier n'existe pas on dit que l'élément est d'ordre infini. Un élément d'ordre fini est dit également de torsion. Si sur une structure algébrique (en particulier sur un groupe) nous disposons d'une relation d'ordre, le cône positif désigne l'ensemble des éléments positifs de cette structure.

\section{Group-nets}

\subsection{Strict-group-nets}

\subsubsection{Motivation initiale, définitions et premiers résultats}

Il est assez courant qu'une même action permette de passer d'un état à un autre et vice versa. Actionner une lampe via un bouton poussoir par exemple, permet indifféremment de l'allumer ou de l'éteindre. La figure 2 montre le système de transitions associé.

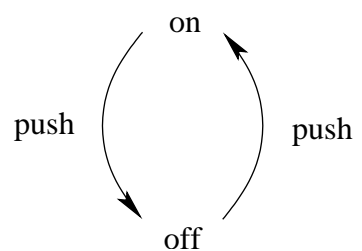

Figure 2. Action d'un bouton poussoir

Il n'existe aucun réseau de Petri dont le graphe des marquages est isomorphe à ce système de transitions étiqueté, car tous les éléments non nul de l'algèbre des places d'un réseau de Petri sont d'ordre infini. On pourrait bien entendu le faire à l'aide d'un réseau de Petri étiqueté avec au moins deux transitions distinctes portant la même étiquette et au moins deux places. L'idée de travailler sur un groupe contenant des éléments d'ordre 2 pourrait permettre de n'utiliser qu' une seule transition et qu'une seule 
place. Si on considère un système plus complexe avec des cycles de longueurs différentes, chacun étiqueté par une seule étiquette, le nombre de transitions et de places nécessaires dans un réseau de Petri étiqueté augmentera en regard de la complexité du système. Dans le cadre de la vérification de systèmes parallèles et distribués, l'obtention de modèles compacts peut s'avérer cruciale. (Juhás, 1998) dans sa thèse propose d'utiliser des algèbres partielles permettant de restreindre ainsi la règle de tir, l'algèbre des réseaux de Petri étant vue comme cône positif du groupe des entiers relatifs. Mais, pour un groupe cyclique fini, la manière de restreindre la loi devient a priori arbitraire (car notamment, il n'est pas possible d'y définir un ordre compatible avec la loi du groupe). Une solution simple car universelle consiste à interdire le zéro du groupe. Reste alors une question : pourquoi continuer à tirer une transition en deux temps (phase de consommation et phase de production), sachant qu'il n'est plus nécessaire d'orienter le graphe car tout élément est inversible? Nous proposons donc de définir une nouvelle classe de réseaux appelée strict-group-nets de la manière suivante :

Définition 3.1 (Strict-group-nets) Soit $(G,+, 0)$ un groupe (non nécessairement abélien) de cardinal au moins 2 et d'élément neutre 0 . Un strict-group-net sur G est un quadruplet $\left(P, T, f, M_{0}\right)$ où $P$ et $T$ sont des ensembles finis disjoints (respectivement l'ensemble des places et des transitions), $f: P \times T \longrightarrow G$ est la fonction de poids et $M_{0}: P \longrightarrow G \backslash\{0\}$ est le marquage initial. Plus généralement, toute application de $P$ dans $G \backslash\{0\}$ est appelée un marquage. Par souci de concision un strict-group-net sur $G$ sera également noté strict-G-net, les strict-group-nets désignant la classe des strict-G-nets pour tout groupe $G$.

Définition 3.2 (Règle de tir pour les strict-group-nets) Soit $N=\left(P, T, f, M_{0}\right)$ un strict-group-net. Une transition $t \in T$ est franchissable à partir du marquage $M$ si $\forall p \in P, M(p)+f(p, t) \neq 0$. Dans ce cas, le nouveau marquage $M^{\prime}$ obtenu après le tir de t est défini par $\forall p \in P, M^{\prime}(p)=M(p)+f(p, t)$. On dénote cette transition $\operatorname{par} M[t\rangle M^{\prime}$.

Il faut faire attention à l'ordre de composition de $M(p)$ et de $f(p, t)$ si la loi du groupe est non commutative.

La figure 3 propose une solution au problème du bouton poussoir avec une place et une transition alors que la figure 4 expose dans sa partie gauche une solution basée sur l'utilisation du groupe produit $\mathbb{Z} / 6 \mathbb{Z} \times \mathbb{Z} / 12 \mathbb{Z}$ à un problème de modélisation du comportement, présenté à droite de la figure, composé de deux cycles de longueurs différentes. 


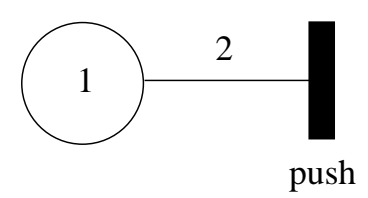

Figure 3. Strict- $\mathbb{Z} / 4 \mathbb{Z}$-net modélisant l'action d'un bouton poussoir
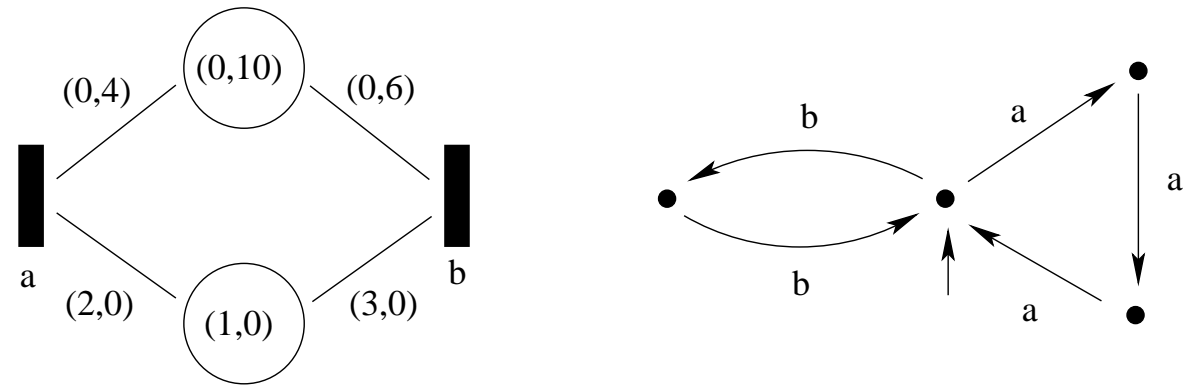

Figure 4. Strict- $\mathbb{Z} / 6 \mathbb{Z} \times \mathbb{Z} / 12 \mathbb{Z}$-net modélisant un comportement composé de deux cycles imbriqués

Un des grands intérêts des strict-group-nets est qu'ils permettent de retrouver les réseaux élémentaires.

Proposition 3.1 Les strict- $\mathbb{Z} / 3 \mathbb{Z}$-nets sont les réseaux élementaires.

Cette proposition découle directement de l'isomorphisme des types des strict$\mathbb{Z} / 3 \mathbb{Z}$-nets et des réseaux élementaires. Les outils utilisés à cette fin sont ceux de la synthèse de réseaux en général (le lecteur pourra trouver en particulier dans (Badouel et al., 1999) tout ce qui concerne les notions de région et de type d'un réseau).

On a montré dans (Guillou, 2004) que les strict- $\mathbb{Z} / p \mathbb{Z}$-nets sont des extensions strictes des strict- $\mathbb{Z} / 3 \mathbb{Z}$-nets pour $p>3$ et que si $p$ et $q$ sont strictement plus grands que 3 et différents, strict- $\mathbb{Z} / p \mathbb{Z}$-nets et strict- $\mathbb{Z} / q \mathbb{Z}$-nets sont incomparables. Enfin, strict- $\mathbb{Z} / p \mathbb{Z}$-nets et strict- $\mathbb{Z}$-nets sont également incomparables.

\subsection{2. strict- $\mathbb{Z}-$ nets}

A première vue les réseaux de Petri et les strict- $\mathbb{Z}$-net semblent proches, le théorème suivant permet de commencer à les situer, en donnant notamment l'algorithme qui permet de passer des réseaux de Petri purs aux strict- $\mathbb{Z}$-nets. 
Théorème 3.1 Les RdP purs sont strictement inclus dans les strict-ZZZ-nets.

Preuve Commençons par donner la construction qui permet de passer d'un RdP pur à un strict- $\mathbb{Z}$-net. Soit $R=\left(P, T, f, M_{0}\right)$ un réseau de Petri pur. Pour une place $p \in P$ donnée on pose $n_{p}=\max _{t \in T}\{f(p, t)\}$. Si $n_{p}>0$ on construit $n_{p}$ places $p_{1}, p_{2}, \ldots, p_{n_{p}}$ marquées respectivement par $M_{0}(p)+1, M_{0}(p)+2, \ldots, M_{0}(p)+n_{p}$. Soit $P^{\prime}$ l'ensemble des places ainsi construit lorsque l'on parcourt $P$. Soit $f^{\prime}: P^{\prime} \times T \longrightarrow$ $\mathbb{Z}$ la fonction de flot définie par $f^{\prime}\left(p_{i}, t\right)=-f(p, t)+f(t, p)$. Le strict- $\mathbb{Z}$-net associé est alors $R^{\prime}=\left(P^{\prime}, T, f^{\prime}, M_{0}^{\prime}\right)$ avec $M_{0}^{\prime}$ le marquage initial défini ci-dessus.

La correspondance $\sigma$ entre les marquages de $R$ et de $R^{\prime}$ est la suivante : un marquage $M$ de $R$ est envoyé sur le marquage $M^{\prime}$ de $R^{\prime}$ défini par $M^{\prime}\left(p_{i}\right)=M(p)+i$ avec $1 \leq i \leq n_{p}$, par conséquent $M^{\prime}>0$. Montrons que la correspondance est préservée par le tir des transitions. Soit $M$ un marquage de $R$ et $t$ une transition franchissable à partir de $M$. Par définition pour tout $p \in P, f(p, t) \leq M(p)$ et $M_{1}(p)$ le marquage obtenu après tir de $t$ vaut $M(p)-f(p, t)+f(t, p)$. On a $\left(\sigma\left(M_{1}\right)\right)\left(p_{i}\right)=M_{1}(p)+i=M(p)-f(p, t)+f(t, p)+i=M^{\prime}\left(p_{i}\right)-f(p, t)+f(t, p)$, avec $M^{\prime}=\sigma(M)$. Comme $M_{1}(p)+i>0, M^{\prime}\left(p_{i}\right)-f(p, t)+f(t, p)>0$ et c'est bien le marquage obtenu par le tir de $t$ dans $R^{\prime}$ à partir de $M^{\prime}$.

Maintenant si, pour un marquage $M$ de $R, \sigma(M)[t\rangle M_{1}^{\prime}$ dans $R^{\prime}$ alors, si on note $M^{\prime}=\sigma(M)$, pour toute place $p_{i}$ de $P^{\prime}, M_{1}^{\prime}\left(p_{i}\right)=M^{\prime}\left(p_{i}\right)-f(p, t)+f(t, p)=$ $M(p)+i-f(p, t)+f(t, p) \neq 0$ quel que soit $i$ entre 1 et $n_{p}$. Comme $n_{p}=$ $\max _{t \in T}\{f(p, t)\}$ nécessairement $M_{1}^{\prime}>0$. Donc $M(p)+i-f(p, t)+f(t, p)>0$ pour $0 \leq i \leq n_{p}$ et, par conséquent, $M(p)-f(p, t)+f(t, p) \geq 0$. Le réseau étant pur, $t$ est franchissable à partir de $M$ et $M_{1}$ le marquage obtenu vaut $M_{1}(p)=M(p)-f(p, t)+f(t, p)$. On constate bien que $\sigma\left(M_{1}\right)=M_{1}^{\prime}$.

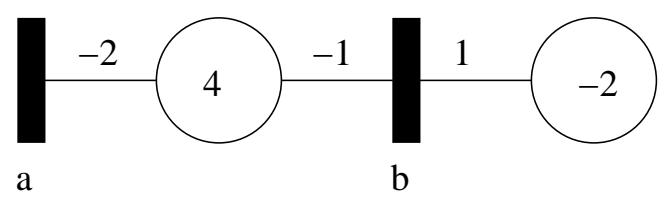

Figure 5. Strict-ZZ-net dont le langage ne peut être celui d'un RdP

Enfin l'inclusion est stricte car le langage associé au strict- $\mathbb{Z}$-net de la figure 5 est l'ensemble des préfixes de $b a^{*}+a b a^{*}$ et ne peut pas être le langage d'un RdP, même impur. En effet, dans un RdP, pouvoir faire $a^{*}$ (après $b$ ) imposerait que $a$ produise au moins autant de ressources qu'il en consomme. Or, il est possible dès le départ de tirer $a$, on devrait donc pouvoir le tirer de nouveau, mais aa n'appartient pas au langage. $\diamond$

$\mathrm{Du}$ point de vue expressivité et décidabilité il est important de situer les strict- $\mathbb{Z}$-nets par rapport aux réseaux de Petri. 
Théorème 3.2 Strict- $\mathbb{Z}$-nets et RdP sont incomparables.

Preuve D'une part l'exemple précédent (cf. figure 5) montre qu'il existe des strict-ZZ-nets dont le graphe des marquages n'est isomorphe au graphe d'aucun RdP. D' autre part, dans le RdP de la figure 6, l'action de $t$ correspond à l'action de l'élément neutre dans un strict- $\mathbb{Z}$-net. Par conséquent, après le tir de $u$ rien ne devrait interdire le tir de $t$ dans le strict- $\mathbb{Z}$-net correspondant. $\diamond$
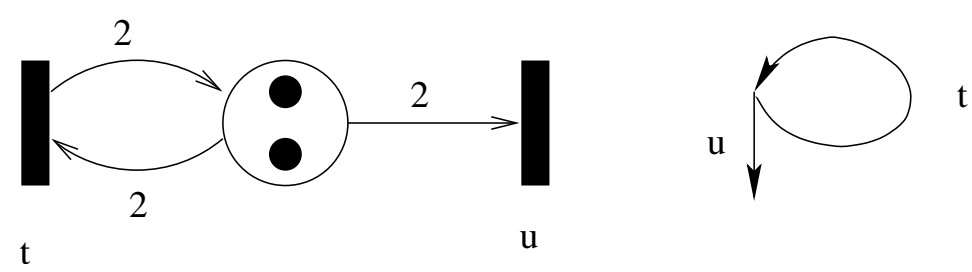

Figure 6. RdP non modélisable par strict- $\mathbb{Z}-n e t$

Malgré cela, nous allons montrer qu'il est possible de définir un arbre et un graphe de couverture, dans un sens plus faible que pour les RdP, rendant décidables les questions de savoir si un réseau est borné et si une place donnée est bornée. Pour cela nous avons besoin d'un ordre bien fondé sur $\mathbb{Z}^{P}$. La définition suivante nous en fournit un.

Définition 3.3 ( $\subseteq$ et $\supseteq$ ) Soient $x$ et y deux éléments de $\mathbb{Z}^{P}$, on écrira $y \supseteq x$ ou $x \subseteq y$ si $\forall p \in P, y(p) \geq x(p) \geq 0 \vee y(p) \leq x(p) \leq 0$. $\subseteq$ est un ordre partiel bien fondé sur $\mathbb{Z}^{P}$.

La définition suivante sera utile pour le prochain théorème.

Définition 3.4 (Séquence sans saut à partir d'un marquage $M$ ) Une séquence $\sigma=t_{1} t_{2} \ldots t_{n}(n \geq 0)$ est une séquence sans saut à partir de $M$ si les composantes des marquages atteints à partir de $M$ par franchissement de $\sigma$ ne changent pas de signe.

Le résultat suivant est un classique des RdP, son adaptation aux strict- $\mathbb{Z}$-nets présente un piège.

Théorème 3.3 Soit $\sigma$ une séquence infinie d'un strict- $\mathbb{Z}-n e t$, alors on peut extraire de $\sigma$ une sous-séquence finie qui soit une séquence répétitive.

Preuve Soit $a_{1} a_{2} a_{3} \ldots$ une séquence infinie et $\left(M_{i}\right)_{i \in \mathbb{N}}$ la suite (infinie) des marquages associés $M_{0}\left[a_{1}\right\rangle M_{1}\left[a_{2}\right\rangle M_{2} \ldots$. On notera $M(j)$ la $j$ ième composante du marquage $M$ (i.e. on voit $M$ comme un vecteur plutôt que comme une fonction). On sépare en deux sous-ensembles $C_{1}^{1}$ et $C_{2}^{1}$ les indices des marquages :

$$
C_{1}^{1}=\left\{i \mid M_{i}(1)>0\right\} \quad C_{2}^{1}=\left\{i \mid M_{i}(1)<0\right\}
$$


Au moins un de ces deux ensembles est infini, on peut supposer sans perte de généralité qu'il s'agit de $C_{1}^{1}$. On recommence le procédé sur la deuxième composante :

$$
C_{1}^{2}=\left\{i \mid i \in C_{1}^{1} \text { et } M_{i}(2)>0\right\} \quad C_{2}^{2}=\left\{i \mid i \in C_{2}^{1} \text { et } M_{i}(2)<0\right\}
$$

et ainsi de suite jusqu'à la $|P|^{\text {ième }}$ composante.

On obtient donc un ensemble infini d'indices $C_{k}^{|P|}(k=1$ ou 2$)$ pour lequel les marquages correspondant ont toutes leurs projections sur une composante donnée de même signe. Appelons $\left(M_{i}^{\prime}\right)_{i \in \mathbb{N}^{*}}$ la suite $\left(M_{i}\right)_{i \in C_{k}^{|P|}}$. De la même manière que de toute suite de $\mathbb{N}^{P}$ on peut extraire une suite infinie croissante (pour l'ordre naturel), il est possible d'extraire de $\left(M_{i}^{\prime}\right)_{i \in \mathbb{N}^{*}}$ une suite infinie croissante au sens de $\subseteq$. Notons $\left(M_{i}^{c}\right)_{i \in \mathbb{N}^{*}}$ cette suite. Malheureusement, rien ne dit que la séquence entre les marquages $M_{1}^{c}$ et $M_{2}^{c}$ est une séquence répétitive. En effet, si nous observons le réseau ci-dessous :

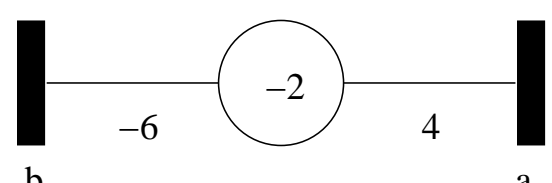

on s'aperçoit qu'après $-2[a\rangle 2[b\rangle-4$ on ne peut plus faire $a b$. Il reste à imposer aux composantes de ne pas changer de signe dans les marquages obtenus lors de la séquence de franchissements.

Dans $\left(M_{i}^{c}\right)_{i \in \mathbb{N}^{*}}$ un certain nombre de places sont bornées. Notons $P_{1}$ l'ensemble de ces places et $P_{2}$ son complémentaire. On extrait de $\left(M_{i}^{c}\right)_{i \in \mathbb{N}^{*}}$ la suite $\left(O_{i}\right)_{i \in \mathbb{N}^{*}}$ dont les composantes selon $P_{1}$ sont constantes (et égales au max ou au min de leurs valeurs). En particulier on note $\sigma_{0}$ la séquence qui permet de passer de $O_{1}$ à $O_{2}$.

Intéressons-nous maintenant aux places de $P_{2}$. Pour $p \in P_{2}$, si $O_{1}(p)>0$, comme $\left(O_{i}(p)\right)_{i \in \mathbb{N}^{*}}$ est non bornée, $\exists j_{p} \in \mathbb{N}^{*}$ tel que $\forall k \geq j_{p}, O_{k}(p)>$ $\left|\sigma_{0}\right| * \max _{t \in T}\{-f(p, t)\}$, où $\left|\sigma_{0}\right|$ représente la longueur de la séquence $\sigma_{0}$. De même si $O_{1}(p)<0, \exists j_{p} \in \mathbb{N}^{*}$ tel que $\forall k \geq j_{p}, O_{k}(p)<-\left|\sigma_{0}\right| * \max _{t \in T}\{f(p, t)\}$. On prend $d=\max \left\{j_{p} \mid p \in P_{2}\right\}$ et à partir du marquage $O_{d}, \sigma_{0}$ est croissante sans saut, donc répétitive. $\diamond$

Nous sommes en mesure maintenant de donner l'algorithme de construction de l'arbre de couverture d'un $\mathbb{Z}$-net. Nous notons $\mathbb{Z}^{\infty}=\mathbb{Z} \cup\{-\infty,+\infty\}$. L'ordre usuel de $\mathbb{Z}$ est étendu à $\mathbb{Z}^{\infty}$ par les conditions $-\infty<n<+\infty$ pour tout entier relatif $n$ et nous posons $w \pm n=w$ avec $w \in\{+\infty,-\infty\}$ et $n \in \mathbb{Z}$. L'ordre $\subseteq$ est également étendu de manière naturelle à $\left(\mathbb{Z}^{\infty}\right)^{P}$.

Définition 3.5 (Arbre et graphe de couverture) Soit $N=\left(P, T, f, M_{0}\right)$ un strictgroup-net. L'arbre de couverture de $N$, noté $A C(N)$, est un arbre où l'ensemble des sommets $S$ est étiqueté par $\left(\mathbb{Z}^{\infty}\right)^{P}$ (étiquettes appelées «marquages généralisés») 
et l'ensemble des arcs par T. AC $(N)$ est défini récursivement par l'application des règles de construction suivantes :

1. La racine $r$ de $A C(N)$ est étiquetée par $M_{0}$.

2. Soit s un sommet déjà construit de $A C(N)$ et étiqueté par $q \in\left(\mathbb{Z}^{\infty}\right)^{P}$.

a. Si l'étiquette de s est identique à l'étiquette d'un ancêtre de s, alors s n'a pas de fils.

b. Dans le cas contraire, $s$ a un fils $s^{\prime}$ pour chaque transition $t$ telle que $\forall p, f(p, t)+q(p) \neq 0 . L^{\prime}$ arc $\left(s, s^{\prime}\right)$ de $A C(N)$ est étiqueté par $t$, et $s^{\prime}$ est étiqueté par $q^{\prime}$ où l'on définit chaque composante $q^{\prime}(p)$ pour $p \in P$ par:

i. Si, lors du parcours du chemin entre $r$ et $s^{\prime}$, il existe deux sommets distincts $s_{1}$ et $s_{2}$ pris dans l'ordre et si la séquence $w$ entre $s_{1}$ et $s_{2}$ est une séquence strictement croissante (au sens de $\subseteq$ ) sans saut à partir du marquage généralisé $q+f t$ (où $f$ est vue comme la matrice d'incidence du réseau) alors si $q(p)<q(p)+f(p,.) \bar{w}$ (où $\bar{w}$ est l'image commutative de $w)$ on a $q^{\prime}(p)=-\infty$ et si $q(p)>q(p)+$ $f(p,.) \bar{w}$ on a $q^{\prime}(p)=+\infty$

ii. Dans le cas contraire $q^{\prime}(p)=q(p)+f(p,)$.$t .$

Le graphe de couverture de $N$, noté $G C(N)$, est obtenu en identifiant les sommets de même étiquette de $A C(N)$.

REMARQUE. - L'arbre de couverture est bien défini car il ne peut y avoir de conflit lors du passage d'une composante d'une étiquette à l'infini entre $+\infty$ et $-\infty$. En effet, la séquence devant être sans saut, une telle composante ne peut changer de signe et ceci quelle que soit la séquence $w$ choisie.

REMARQUE. - La notion de couverture que l'on vient de donner n'est pas aussi forte que celle que l'on manipule avec les RdP. Il ne s'agit pas en tous cas d'une couverture au sens de $\subseteq$. En effet, soit le réseau avec son graphe de couverture ci-dessous :

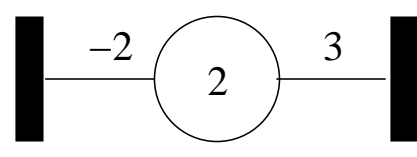

a b

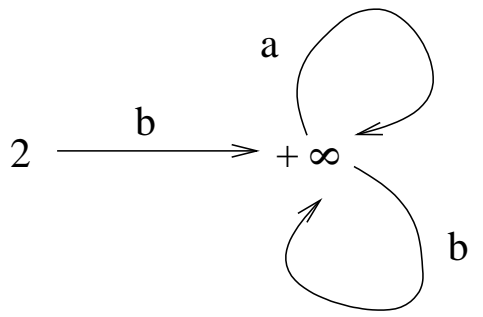

\section{b}

Si on tire la séquence baaa, le marquage obtenu est négatif alors qu'il est couvert par $+\infty$ dans le graphe de couverture.

Soit $q$ l'étiquette d'un sommet d'un arbre de couverture, on note

$$
q^{\infty}=\{p \in P \mid q(p)=+\infty \text { ou } q(p)=-\infty\}
$$


Théorème 3.4 L'arbre de couverture est fini.

Preuve Supposons qu'il soit infini, alors il contient au moins une branche infinie. Soit $\left(q_{i}\right)_{i \geq 0}$ la suite infinie de ses étiquettes. $+\infty$ et $-\infty$ étant héréditaires, il existe $k \in \mathbb{N}$ tel que $\forall i \geq k, q_{i}^{\infty}=q_{k}^{\infty}$ et $\forall p \in q_{k}^{\infty}, q_{i}(p)=q_{k}(p)$. De plus $q_{k}^{\infty} \neq P$ car sinon la branche serait finie. On peut alors extraire une suite strictement croissante $\left(q_{i_{j}}\right)_{j \geq 0}$ avec $i_{0} \geq k$ (strictement croissante au sens de $\subseteq$ car aucune étiquette ne peut se répéter). De la suite $\left(q_{i_{j}}\right)_{j \geq 0}$ on extrait une sous-suite $\left(q_{i}^{\prime}\right)_{i \geq 0}$ telle que une partie des composantes soient constantes et l'autre strictement croissantes. Entre $q_{0}^{\prime}$ et $q_{1}^{\prime}$ on a une séquence strictement croissante et il existe $m \in \mathbb{N}$ tel que cette séquence soit franchissable à partir de $q_{m}^{\prime}$ et strictement croissante sans saut. Ce qui contredit le fait que $q_{m}^{\prime \infty}=q_{k}^{\infty} \cdot \diamond$

La proposition suivante justifie le terme de « couverture».

Propriété 3.1 Soit $\sigma$ une séquence de franchissements telle que $M_{0}[\sigma\rangle M$. Il existe un unique chemin dans $G C(N)$ partant de $M_{0}$ et étiqueté par $\sigma$, l'extrémité q de ce chemin vérifiant $\forall p \in P, q(p)=M(p)$ ou $q(p)=+\infty$ ou $q(p)=-\infty$.

Preuve Par récurrence sur la longueur de $\sigma$. Si $\sigma$ est de longueur 0, c'est le chemin d'origine et d'extrémité $M_{0}$. Supposons la propriété vraie pour toute séquence de franchissements de longueur $n$ avec $n \geq 0$. Prenons une séquence de franchissements $\sigma$ de longueur $n+1$. $\sigma$ peut alors s'écrire sous la forme $\sigma=z t$ avec $z$ de longueur $n$. On a l'exécution suivante $M_{0}[z\rangle M^{\prime}[t\rangle M$. Par hypothèse de récurrence il existe un unique chemin dans $G C(N)$ partant de $M_{0}$ et étiqueté par $z$, l'extrémité $q^{\prime}$ de ce chemin vérifiant $\forall p \in P, q^{\prime}(p)=M^{\prime}(p)$ ou $q^{\prime}(p)=+\infty$ ou $q^{\prime}(p)=-\infty$. Comme $\forall p \in P, M^{\prime}(p)+f(p, t) \neq 0$ le sommet $q^{\prime}$ admet un successeur $q$ avec l'arc joignant ces deux sommets étiqueté par $t$. Les règles de construction de l' $A C$ imposent à $q$ d'avoir les valeurs souhaitées, l'unicité du chemin découlant du caractère déterministe du graphe. $\diamond$

Lemme 3.1 Soit $N$ un strict-ZZ-net. Tout sous-graphe de $G C(N)$ ne contenant pas de $+\infty$ ni de $-\infty$ est un sous-graphe du graphe des marquages de $N$.

Preuve Tant qu'il n'y a ni $+\infty$ ni $-\infty$ la règle de construction de l' $A C(N)$ est la même que celle du graphe des marquages. $\diamond$

Théorème 3.5 (Décidabilité du caractère borné d'un strict-ZZ-net) Soit $N$ un strict-Z-Z-net. $N$ est non borné ssi il existe un sommet $q$ de $G C(N)$ tel que $q^{\infty} \neq \emptyset$.

Preuve Soit $q$ un sommet de $G C(N)$ tel que $q^{\infty} \neq \emptyset$. Dans $A C(N) q$ étiquette un sommet $s$. On peut supposer que $s$ est le premier sommet construit ayant cette propriété. Donc tout sommet $s^{\prime}$ sur le chemin de $r$ à $s$ ( $s$ exclu) est étiqueté par un marquage $M^{\prime}$ accessible et si $w$ est le mot étiquetant le chemin de $r$ à $s^{\prime}$ on a $M[w\rangle M^{\prime}$. 
Il existe sur le chemin de $r$ à $s$ deux sommets $s_{1}$ et $s_{2}$ étiquetés respectivement par $q_{1}$ et $q_{2}$ tels que $q_{1} \subseteq q_{2}$ et le mot qui étiquette le chemin de $s_{1}$ à $s_{2}$ est une séquence strictement croissante et sans saut à partir du marquage $q^{\prime}+f(., t)$ où $q^{\prime}$ est l'étiquette du prédecesseur de $s$ dans l' $A C$ et $t$ l'étiquette de l'arc reliant ce prédecesseur à $s$. $q^{\prime}+f(., t)$ est un marquage accessible. Donc $N$ est non borné.

Soit $N$ non borné, alors il existe une séquence strictement croissante et sans saut. Cette séquence est obtenue avec la construction exhibée dans la preuve du théorème 3.3. Soit $u=w . x$ un mot tel que $x$ soit une séquence strictement croissante et sans saut à partir du marquage atteint après exécution de $w$. On note $M_{0}[w\rangle M[x\rangle M^{\prime}$.

Il existe un unique chemin dans $G C(N)$ partant de $M_{0}$ et étiqueté par $w x$, l'extrémité $q$ de ce chemin vérifiant $\forall p \in P, q(p)=M^{\prime}(p)$ ou $q(p)=+\infty$ ou $q(p)=-\infty$. Supposons que $\forall p \in P, q(p)=M^{\prime}(p)$. $x$ étant une séquence strictement croissante et sans saut à partir du marquage $q$, nécessairement $q^{\infty} \neq \emptyset$. Par conséquent $\exists p \in P$ tel que $q(p)= \pm \infty . \diamond$

Lemme 3.2 Soit $N$ un strict- $\mathbb{Z}$-net et $A C(N)$ son arbre de couverture. Soit s un sommet de $A C(N)$ étiqueté par $q$, alors pour tout $k \in \mathbb{N}$, il existe un marquage $M$ accessible tel que $M(p)=q(p)$ pour tout $p \notin q^{\infty}$, et, pour tout $p \in q^{\infty}, M(p)$ du même signe que $q(p)$ et $|M(p)| \geq k$.

Preuve C'est vrai pour la racine. On suppose que c'est vrai pour un sommet $s_{1}$ de $A C(N)$. Montrons que c'est vrai pour tout successeur $s_{2}$ de $s_{1} . s_{1}$ est étiqueté par $q_{1}$ et $s_{2}$ par $q_{2}$.

On pose $P_{1}=q_{1}^{\infty}$ et $P_{2}=q_{2}^{\infty}$, on a $P_{1} \subset P_{2}$ (hérédité des infinis). On pose enfin $P_{2}=P_{1} \cup P_{3}$ avec $P_{1} \cap P_{3}=\emptyset$.

On appelle $t$ l'étiquette de l'arc joignant $s_{1}$ à $s_{2}$. Si $P_{3}=\emptyset$ prenons un $k \in \mathbb{N}$. Par hypothèse d'induction, il existe un marquage $M$ accessible tel que $\forall p \notin P_{1}, M(p)=$ $q_{1}(p)$ et $\forall p \in P_{1},|M(p)| \geq k+\max _{p^{\prime} \in P}\left\{\left|f\left(p^{\prime}, t\right)\right|\right\}$ et $M(p)$ du même signe que $q_{1}(p)$. Alors $M^{\prime}$ défini par $M[t\rangle M^{\prime}$ vérifie bien les conditions du lemme.

Si $P_{3} \neq \emptyset$, soit $p \in P_{3}$ alors il existe deux sommets distincts $s_{1}^{\prime}$ et $s_{2}^{\prime}$ sur le chemin de $r$ à $s_{2}$ tels que $w_{p}$ la séquence entre $s_{1}^{\prime}$ et $s_{2}^{\prime}$ soit strictement croissante et sans saut (au moins pour $p$ ) à partir du marquage généralisé $q_{1}+C(., t)$.

On va s'arranger pour mettre des valeurs assez grandes (en valeur absolue) dans les places de $P_{1}$ pour pouvoir obtenir ce que l'on veut dans $P_{2}$.

Pour $p^{\prime}$ dans $P_{1}$ on pose $\alpha_{p^{\prime}}=f\left(p^{\prime},.\right) \overline{w_{p}}$ et $\alpha_{p}^{\max }=\max _{p^{\prime} \in P_{1}}\left\{\left|\alpha_{p^{\prime}}\right|\right\}$. On pose enfin $\alpha=\max _{p^{\prime} \in P_{1}}\left\{\left|f\left(p^{\prime},.\right) t\right|\right\}$. Par hypothèse d'induction on peut trouver un marquage $M$ accessible tel que $\left|M\left(p^{\prime}\right)\right| \geq k \sum_{p \in P_{3}} \alpha_{p}^{\max }+k+\alpha$ et $M\left(p^{\prime}\right)$ de même signe que $q_{1}\left(p^{\prime}\right)$ pour $p^{\prime} \in P_{1}$ et pour $p^{\prime} \notin P_{1}, M\left(p^{\prime}\right)=q_{1}\left(p^{\prime}\right)$. Alors le marquage $M_{2}^{\prime}$ défini par $M[t\rangle M_{2}\left[w_{p_{1}}^{k} w_{p_{2}}^{k} \ldots w_{p_{\left|P_{3}\right|}}^{k}\right\rangle M_{2}^{\prime}$, où $p_{1}, p_{2}, \ldots p_{\left|P_{3}\right|}$ sont les places de $P_{3}$, vérifie la propriété demandée. $\diamond$ 
Théorème 3.6 (Décidabilité du caractère borné d'une place d'un strict-ZZ-net) Soit $N$ un strict- $\mathbb{Z}-n e t$. Une place $p$ est non bornée ssi il existe un sommet $q$ de $G C(N)$ tel que $q(p)= \pm \infty$.

Preuve Soit $N$ un strict- $\mathbb{Z}$-net qui possède une place $p$ non bornée. Pour tout $k \in \mathbb{N}$, il existe un chemin dans le $G C$ tel que l'étiquette $q$ de l'extrémité de ce chemin vérifie $|q(p)| \geq k$. Réciproquement, le lemme précédent permet de conclure. $\diamond$

\subsection{Group-nets}

Une manière classique d'augmenter l'expressivité d'une classe de réseaux consiste à lui adjoindre des arcs particuliers, c'est le cas par exemple des RdP avec arcs inhibiteurs ou avec arcs Reset. Dans l'optique d'obtenir une extension des RdP nous définissons les group-nets comme étant les strict-group-nets munis d'un arc inconditionnel qui autorise une marque à prendre la valeur de l'élément neutre du groupe.

\subsubsection{Définitions}

Définition 3.6 (Group-net) Soit $G$ un groupe. Un group-net sur $G$ est un quadruplet $N=\left(P, T, f, M_{0}\right)$ où $P$ est l'ensemble fini des places, $T$ est l'ensemble fini des transitions (disjoint de $P$ ) et $f$ est la relation de flot, c'est-à-dire une application de $P \times T$ vers $\{0,1\} \times G$. Un arc est une paire $(p, t) \in d o m(f)$. L'arc $(p, t)$ est dit conditionnel si $\pi_{1}(f(p, t))=0$, inconditionnel si $\pi_{1}(f(p, t))=1$ où $\pi_{1}$ est la première projection. $M_{0} \in G^{P}$ est le marquage initial du réseau. Comme pour les strict-group-nets un group-net sur G sera noté G-net et les group-nets de manière générale désignent la classe des $G$-nets pour tout groupe $G$.

Définition 3.7 (Règle de tir pour les group-nets) Soit $N=\left(P, T, f, M_{0}\right)$ un groupnet sur $G, t \in T$ est franchissable à partir de $M \in G^{P}$ si pour tout $p \in P$, $\pi_{1}(f(p, t))=0 \Longrightarrow M(p)+\pi_{2}(f(p, t)) \neq 0$ où $\pi_{1}$ est la première projection et $\pi_{2}$ la seconde. Dans le cas où $t$ est franchissable à partir de $M$, le tir de t produit un nouveau marquage $M^{\prime}$ défini par $M^{\prime}(p)=M(p)+\pi_{2}(f(p, t))$ pour toute place $p$ de $P$.

La figure 7 montre un exemple typique de $\mathbb{Z}$-net, les arcs inconditionnels apparaissant comme des arcs doubles. Dans cet exemple, $b$ ne peut être tiré, mais $a$ et $c$ le peuvent.

\subsection{2. $\mathbb{Z}$-nets}

Le théorème suivant confirme que les $\mathbb{Z}$-nets étendent bien les $\mathrm{RdP}$.

Théorème 3.7 Les $\mathbb{Z}$-nets sont une extension des $R d P$. 


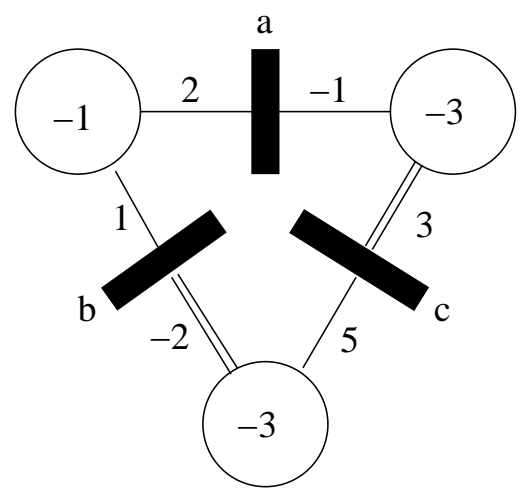

Figure 7. Un $\mathbb{Z}$-net

Preuve Soit $N=\left(P, T, f, M_{0}\right)$ un réseau de Petri. Pour tout $p \in P$, soit $E_{p}=$ $\cup_{t \in T}\left\{M_{0}(p)-f(t, p)+1, M_{0}(p)-f(t, p)+2, \ldots, M_{0}(p)-f(t, p)+f(p, t)\right\}$. Pour tout $p \in P$ et pour chaque $i \in E_{p}$, on définit une place $p_{i}$. Soit $P^{\prime}$ l'ensemble des places définies ainsi en faisant parcourir $P$ à $p$. Le marquage initial de chaque place $p_{i} \in P^{\prime}$ est son indice $i$. Soit $f^{\prime}: P^{\prime} \times T \longrightarrow \mathbb{Z}$ la relation de flot définie ainsi : pour tout $p \in P$ et pour chaque $i \in E_{p}$, on pose $f^{\prime}\left(p_{\alpha}, t\right)=(0,-f(p, t)+f(t, p))$ si $M_{0}(p)-$ $f(t, p)+1 \leq \alpha \leq M_{0}(p)-f(t, p)+f(p, t)$ et $f^{\prime}\left(p_{\alpha}, t\right)=(1,-f(p, t)+f(t, p))$ sinon. Il suffit ensuite de vérifier que le graphe des marquages de $N^{\prime}=\left(P^{\prime}, T, f^{\prime}, M_{0}^{\prime}\right)$ est isomorphe à celui de $N . \diamond$

D'après la figure 5, l'inclusion est stricte car les $\mathbb{Z}$-nets étendent les strict- $\mathbb{Z}$-nets.

Il se trouve que la définition et les propriétés de l'arbre de couverture pour les strict- $\mathbb{Z}$-nets restent valides pour les $\mathbb{Z}$-nets. Par conséquent :

Théorème 3.8 (Décidabilité du caractère borné d'une place et d'un $\mathbb{Z}$-net) Soit $N$ un $\mathbb{Z}$-net. Le problème de savoir si une place donnée de $N$ est bornée est décidable ainsi que le problème de savoir si $N$ lui-même est borné.

Beaucoup d'extensions strictes des RdP, tels les réseaux avec arcs inhibiteurs, ont la puissance des machines de Turing. La figure 8 exhibe un réseau avec arcs inhibiteurs qui ne peut-être modélisé à l'aide d'un $\mathbb{Z}$-net. En effet, si un tel réseau existait, l'action de $a$ correspondrait à l'action de l'élément neutre du groupe et par conséquent $a$ serait tirable après un ou deux tirs de $b$ (pour tenir compte du fait que $b$ pourrait être reliée par un arc inconditionnel) dont l'action n'est pas celle d'un élément neutre; ce qui n'est pas le cas du réseau de la figure 8. Réciproquement la figure 5 donne un $\mathbb{Z}$-net qui ne possède pas de réseau avec arcs inhibiteurs équivalent. $\mathbb{Z}$-nets et réseaux de Petri avec arcs inhibiteurs sont incomparables ce qui laisse ouvertes des questions comme l'accessibilité pour les $\mathbb{Z}$-nets. De nombreux problèmes de décidabilité sont attaqués 
par le biais des machines à compteur ou encore des systèmes d'addition de vecteurs avec états (VASS) (introduits dans (Hopcroft et al., 1979)) qui sont équivalents aux réseaux de Petri. Par rapport aux VASS, le comportement des $\mathbb{Z}$-nets est fondamentalement différent, car, géométriquement parlant, les séquences de tirs possibles ne sont plus limitées à un espace défini comme une intersection de demi-espaces (liés au fait que le nombre de marques dans une place doit rester supérieur ou égal à zéro) mais doivent simplement éviter des hyperplans (liés au fait qu'une place ne peut prendre la valeur 0). Les techniques utilisables semblent donc très différentes.

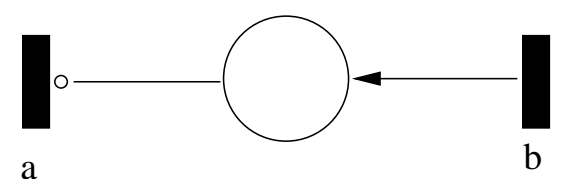

Figure 8. Réseau avec arc inhibiteur non modélisable par $\mathbb{Z}$-net.

\subsection{3. $\mathbb{Z} / n \mathbb{Z}-$ nets}

Les $\mathbb{Z} / 2 \mathbb{Z}$-nets jouent un rôle particulier. D'une part il est possible de synthétiser le type des réseaux flip-flop (Schmitt, 1996) par un $\mathbb{Z} / 2 \mathbb{Z}$-net (voir (Guillou, 2003)), c'est-à-dire de construire un $\mathbb{Z} / 2 \mathbb{Z}$-net dont le graphe des marquages est isomorphe au type des réseaux flip-flop (qui est lui-même un graphe par définition), par conséquent les $\mathbb{Z} / 2 \mathbb{Z}$-nets constituent une extension (il est facile de montrer qu'elle est même stricte) des réseaux flip-flop. Comme pour ces derniers il est possible de donner un algorithme de synthèse de complexité polynomiale pour les $\mathbb{Z} / 2 \mathbb{Z}$-nets (Guillou, 2003). Par ailleurs le type des $\mathbb{Z} / 2 \mathbb{Z}$-nets (cf. figure 9) est extrêmement général dans l'ensemble des types des réseaux à places binaires. En effet, nous disposons à la fois d'un événement $(1,1)$ permettant de switcher d'un état à l'autre (principe du flip-flop), d'un événement $(0,1)$ induisant un changement d'état mais valide à partir d'un seul état (comme dans les réseaux élémentaires), d'un événement $(1,0)$ jouant le rôle d'un élément neutre et d'un événement $(0,0)$ du même genre mais seulement valide à partir d'un seul des deux états. En particulier, les $\mathbb{Z} / 2 \mathbb{Z}$-nets étendent les réseaux de Petri 1-sauf.

De manière générale, les $\mathbb{Z} /(n+1) \mathbb{Z}$-nets étendent les réseaux de Petri $n$-bornés. En effet, il suffit de reprendre la construction du théorème 3.7, permettant de passer d'un $\mathrm{RdP}$ à un $\mathbb{Z}$-net, en manipulant chaque valeur en tant que classe de congruence modulo $(n+1)$ (on travaille directement dans $\mathbb{Z} /(n+1) \mathbb{Z}$ plutôt que dans $\mathbb{Z}$, ce qui est possible puisque le réseau de départ est réputé $n$-borné) pour obtenir un $\mathbb{Z} /(n+1) \mathbb{Z}$ net. La construction d'un $\mathbb{Z} /(n+1) \mathbb{Z}$-net ayant un comportement cyclique d'ordre $(n+1)$ (par exemple une place marquée par 0 et une transition reliée à la place par un arc inconditionnel étiqueté par 1) permet de se convaincre que cette inclusion est stricte car aucun élément non nul de $\mathbb{Z}$ n'est d'ordre fini. Enfin, $\mathbb{Z} /(n+1) \mathbb{Z}$ est le plus petit groupe, du point de vue de son cardinal, dans l'ensemble des $\mathbb{Z} / m \mathbb{Z}$ qui étend les réseaux de Petri $n$-bornés. 


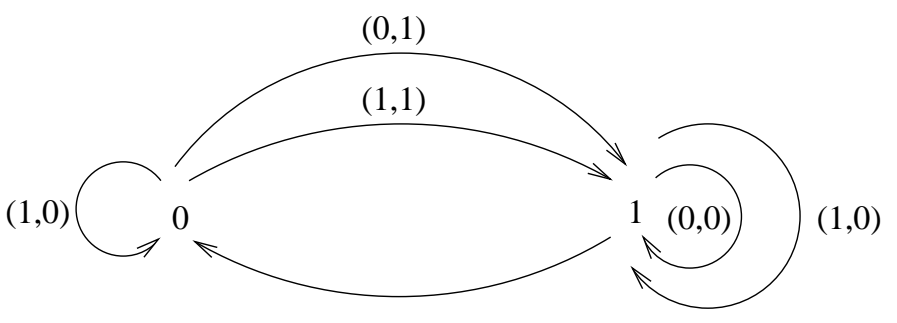

$(1,1)$

Figure 9. Type des $\mathbb{Z} / 2 \mathbb{Z}$-nets

La figure 10 résume l'état de notre classification des classes de group-nets que nous avons étudiées par rapport à un certain nombre de classes de réseaux de Petri usuelles. Les strict- $\mathbb{Z} / 2 \mathbb{Z}$-nets qui sont les $\{0\}$-nets, réseaux dont les graphes de marquages se résument à un unique sommet avec un nombre arbitraire de boucles, apparaissent en tant qu'objet terminal dans la catégorie des systèmes de transitions. Les inclusions y sont strictes et les classes incomparables si aucun chemin ne les relie.

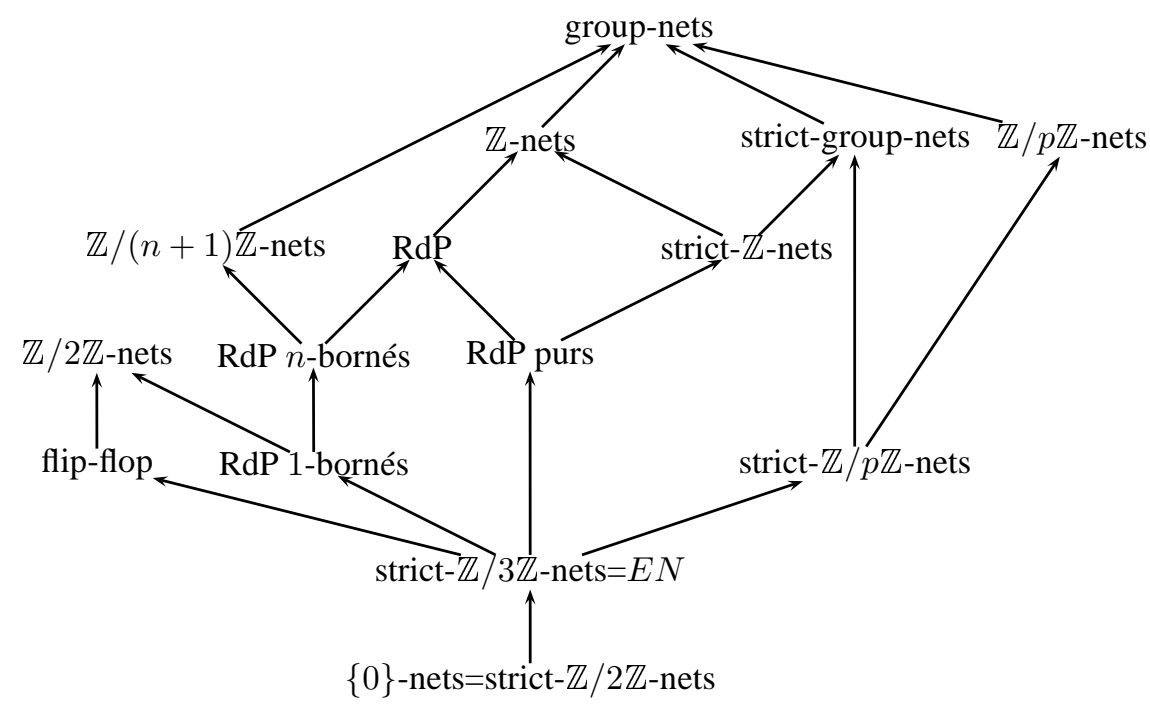

Figure 10. Group-nets et réseaux de Petri

\subsection{Group ${ }_{2}$-nets et Group ${ }_{n}$-nets}

Le choix de ne pas orienter les représentations des group-nets était guidé par l'existence d'inverses dans un groupe. Ainsi, dans $\mathbb{Z}$, consommer de la ressource revient à 
ajouter un élément négatif. Cependant la notion de ressource dans les $\mathbb{Z}$-nets, si elle existe, semble étrange et nous allons voir que d'imposer une règle de tir en deux temps (consommation puis production) comme dans les réseaux de Petri conduit étonnamment à une vision unifiée des $\mathbb{Z}$-nets et des strict- $\mathbb{Z}$-nets.

\subsubsection{Group 2 -nets}

Soit $(G,+, 0)$ un groupe non trivial, d'élément neutre 0 .

Définition 3.8 ( $G_{2}$-net) $U n G_{2}$-net est un quintuplet $N=\left(P, T\right.$, Pré, Post, $\left.M_{0}\right)$ où $P$ est un ensemble fini de places, $T$ est un ensemble fini de transitions disjoint de $P$, Pré et Post sont des applications de $P \times T$ dans $G$ et $M_{0}$ est une application de $P$ dans $G \backslash\{0\}$ appelée marquage initial.

Définition 3.9 (Règle de tir pour les $G_{2}$-net) Soit $N=\left(P, T\right.$, Pré, Post, $\left.M_{0}\right)$ un $G_{2}$-net et $M$ un marquage. La transition $t$ de $T$ est tirable ou franchissable à partir de $M$ si $\forall p \in P, M+\operatorname{Pré}(p, t) \neq 0$ et $M+\operatorname{Pré}(p, t)+\operatorname{Post}(p, t) \neq 0$ et , dans ce cas, on obtient un nouveau marquage $M^{\prime}$ défini par $\forall p \in P, M^{\prime}(p)=M(p)+$ $\operatorname{Pré}(p, t)+\operatorname{Post}(p, t)$. Cette étape est notée $M[t\rangle M^{\prime}$.

La figure 11 donne un $\mathbb{Z}_{2}$-net où sont reprises les conventions usuelles aux réseaux de Petri. Par contre, si pour une place $p$ et une transition $t$ données Pré $(p, t)=0 \mathrm{~V}$ $\operatorname{Post}(p, t)=0$, on se contentera de représenter un seul arc non orienté étiqueté par $\operatorname{Pré}(p, t)+\operatorname{Post}(p, t)$. Dans ce réseau $a$ n'est pas tirable (attention, on ne fait que de l'addition) mais $b$ l'est. Après le tir de $b, a$ n'est toujours pas tirable.

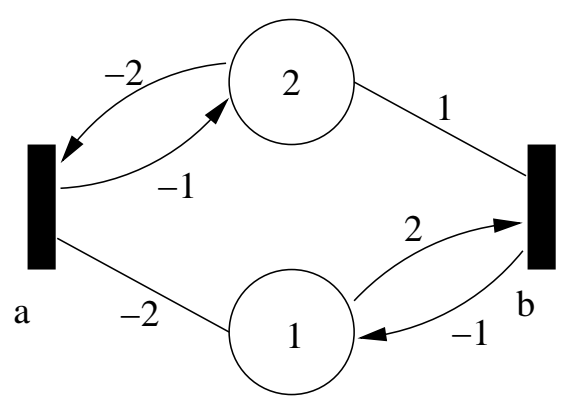

Figure 11. exemple de $\mathbb{Z}_{2}$-net

On a trivialement le résultat suivant :

Propriété 3.2 Tout strict-G-net est un $G_{2}$-net pur. 


\subsection{2. $\mathbb{Z}_{2}$-nets}

Théorème 3.9 Les $\mathbb{Z}_{2}$-nets sont les $\mathbb{Z}$-nets.

Les deux lemmes suivants donnent les algorithmes permettant de construire le $\mathbb{Z}$-net correspondant à un $\mathbb{Z}_{2}$-net donné et réciproquement.

Lemme 3.3 Les $\mathbb{Z}$-nets sont inclus dans les $\mathbb{Z}_{2}$-nets.

Preuve Soit $N=\left(P, T, f, M_{0}\right)$ un $\mathbb{Z}$-net. On définit le $\mathbb{Z}_{2}$-net $N^{\prime}$ de la façon suivante : $N^{\prime}=\left(P, T\right.$, Pré, Post, $\left.M_{0}^{\prime}\right)$ avec $\forall p \in P, M_{0}^{\prime}(p)=2 M_{0}(p)-1$

$\forall p \in P, \forall t \in T$, si $\pi_{1}(f(p, t)=1$ on pose $\operatorname{Pré}(p, t)=0$ et $\operatorname{Post}(p, t)=$ $2 \pi_{2}(f(p, t))$ et si $\pi_{1}\left(f(p, t)=0\right.$,on pose, $\operatorname{Pré}(p, t)=2 \pi_{2}(f(p, t))+$ 1 et $\operatorname{Post}(p, t)=-1$. On termine en montrant que $N$ et $N^{\prime}$ ont leurs graphes des marquages isomorphes. $\diamond$

Lemme 3.4 Les $\mathbb{Z}_{2}$-nets sont inclus dans les $\mathbb{Z}$-nets.

Preuve Soit $N=\left(P, T\right.$, Pré, Post, $\left.M_{0}\right)$ un $\mathbb{Z}_{2}$-net. On construit le $\mathbb{Z}$-net $N^{\prime}=$ $\left(P^{\prime}, T, f, M_{0}^{\prime}\right)$ de la manière suivante : pour tout $p \in P$, pour tout $t \in T, M_{0}^{\prime}(p)=$ $M_{0}(p)$ et $f(p, t)=(0, \operatorname{Pré}(p, t)+\operatorname{Post}(p, t))$, si Pré $(p, t) \neq 0$ et $\operatorname{Post}(p, t) \neq 0$ alors on ajoute une place $p_{t}$ telle que $f\left(p_{t}, t\right)=(0, \operatorname{Pré}(p, t)+\operatorname{Post}(p, t)), M_{0}^{\prime}\left(p_{t}\right)=$ $M_{0}(p)-\operatorname{Post}(p, t)$, et, $\forall t^{\prime} \neq t, f\left(p_{t}, t^{\prime}\right)=\left(1, \operatorname{Pré}\left(p, t^{\prime}\right)+\operatorname{Post}\left(p, t^{\prime}\right)\right) . P^{\prime}$ est défini comme l'union de $P$ et des $\left\{p_{t}\right\}$ ainsi créées. On vérifie ensuite que les graphes des marquages de $N$ et $N^{\prime}$ sont isomorphes. $\diamond$

$\mathbb{Z}$-nets et strict- $\mathbb{Z}$-nets sont donc des cas particuliers de $\mathbb{Z}_{2}$-nets :

Corollaire 3.1 Les strict- $\mathbb{Z}$-nets sont les $\mathbb{Z}_{2}$-nets purs et les $\mathbb{Z}$-nets sont les $\mathbb{Z}_{2}$-nets.

L'existence d'un arbre de couverture pour les $\mathbb{Z}$-nets entraîne le corollaire suivant.

Corollaire 3.2 Pour les $\mathbb{Z}_{2}$-nets les problèmes de la terminaison, du caractère borné d'un réseau et du caractère borné d'une place sont décidables.

Le théorème suivant découle directement des résultats précédents, cependant la preuve s'appuie sur la construction directe d'un $\mathbb{Z}_{2}$-net équivalent à un $\mathrm{RdP}$ donné.

Théorème 3.10 Les $\mathbb{Z}_{2}$-nets sont une extension stricte des réseaux de Petri.

Preuve Conséquence immédiate du fait que les $\mathbb{Z}_{2}$-nets sont les $\mathbb{Z}$-nets. Nous donnons ci-après une manière de construire un $\mathbb{Z}_{2}$-net à partir d'un RdP sans passer par un $\mathbb{Z}$-net. 
Soit $N=\left(P, T, f, M_{0}\right)$ un réseau de Petri marqué. Pour chaque place $p \in P$, on pose $n_{p}=\max _{t \in T}\{f(p, t)\}$. On construit alors $n_{p}$ places appelées $p_{1}, p_{2}, \ldots, p_{n_{p}}$ et marquées respectivement par $M_{0}(p)+1, M_{0}(p)+2, \ldots, M_{0}(p)+n_{p}$. Soit $P^{\prime}$ l'ensemble des places ainsi construites lorsqu'on parcourt $P$. On définit $N^{\prime}=$ $\left(P^{\prime}, T\right.$, Pré, Post, $\left.M_{0}^{\prime}\right)$ le $\mathbb{Z}_{2}$-net pour lequel $M_{0}^{\prime}$ le marquage initial a été donné cidessus et $\forall p_{i} \in P^{\prime}, \forall t \in T, \operatorname{Pré}\left(p_{i}, t\right)=-f(p, t)$ et $\operatorname{Post}\left(p_{i}, t\right)=f(t, p)$. $\diamond$

\subsection{3. $G_{2}$-nets et $G$-nets}

On se pose maintenant la question naturelle : quels sont les liens entre les $G_{2}$-nets et les $G$-nets pour un groupe arbitraire $G$ ?

\section{Théorème 3.11 Les $G_{2}$-nets sont inclus dans les $G$-nets.}

Preuve La construction décrite dans la preuve du lemme 3.4 permet d'associer un $G$-net équivalent à un $G_{2}$-net donné. $\diamond$

La réciproque est évidemment fausse : prenons pour $G$ le groupe à deux éléments $\mathbb{Z} / 2 \mathbb{Z}$. Dans un tel groupe les $(\mathbb{Z} / 2 \mathbb{Z})_{2}$-nets ne peuvent changer d'état ce qui n'est évidemment pas le cas des $\mathbb{Z} / 2 \mathbb{Z}$-nets. Cependant on peut se poser la question suivante : soit un $G$-net donné, existe-t-il un groupe $G^{\prime}$ tel qu'il est possible de construire un $G_{2}^{\prime}$-net équivalent?

Proposition 3.2 Soit $G$ un groupe, alors à tout $G$-net on peut associer un $(\mathbb{Z} / 2 \mathbb{Z} \times$ $G)_{2}$-net équivalent.

Preuve Il est facile de se convaincre que la construction ci-dessous généralisée à l'ensemble des places du $G$-net (à gauche) donne un $(\mathbb{Z} / 2 \mathbb{Z} \times G)_{2}$ - net équivalent (à droite).
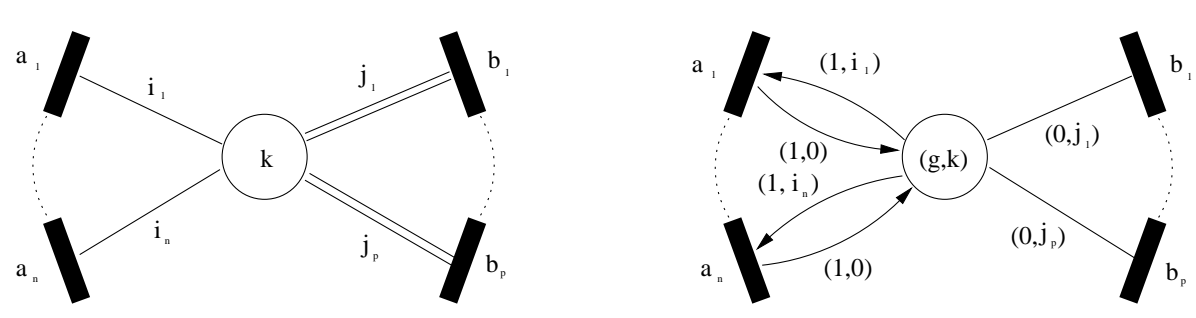

$\diamond$

\subsubsection{Les group $_{n}$-nets}

Dans les réseaux de Petri le franchissement d'une transition se fait en deux temps : il y a tout d'abord consommation de ressource puis production de ressource. Dans 
les group-nets il se fait en un seul temps et on retrouve le franchissement en deux temps dans les group $_{2}$-nets. Pourquoi ne pas essayer de généraliser la règle de franchissement en se donnant la possibilité d'effectuer le franchissement d'une transition en $n$ temps en conservant l'interdiction d'atteindre le zéro du groupe ? On appellera de tels réseaux des group $n_{n}$-nets. En particulier les strict-group-nets sont les group ${ }_{1}$ nets. Nous allons voir que le seul gain effectif de cette généralisation est le passage des group ${ }_{1}$-nets aux group ${ }_{2}$-nets. Pour éviter d'avoir à dessiner $n$ arcs numérotés dans l'ordre d'exécution des $n$ étapes du franchissement d'une transition, on se contentera de n'en représenter qu'un seul étiqueté par un $n$-uplet comportant les $n$ valeurs de flot dans l'ordre. Ainsi les arcs Pré et Post entre une transition $t$ et une place $p$ d'un group ${ }_{2}$-net seront représentés par un seul arc non orienté étiqueté par le couple $(\operatorname{Pré}(p, t), \operatorname{Post}(p, t))$. De manière générale, dans un $\operatorname{group}_{n}$-net, si $n>1$, il suffit de remplacer chaque place par $n-1$ places ayant toutes le marquage de la place d'origine et de les connecter aux transitions pour obtenir deux réseaux équivalents comme le montre la figure ci-dessous.

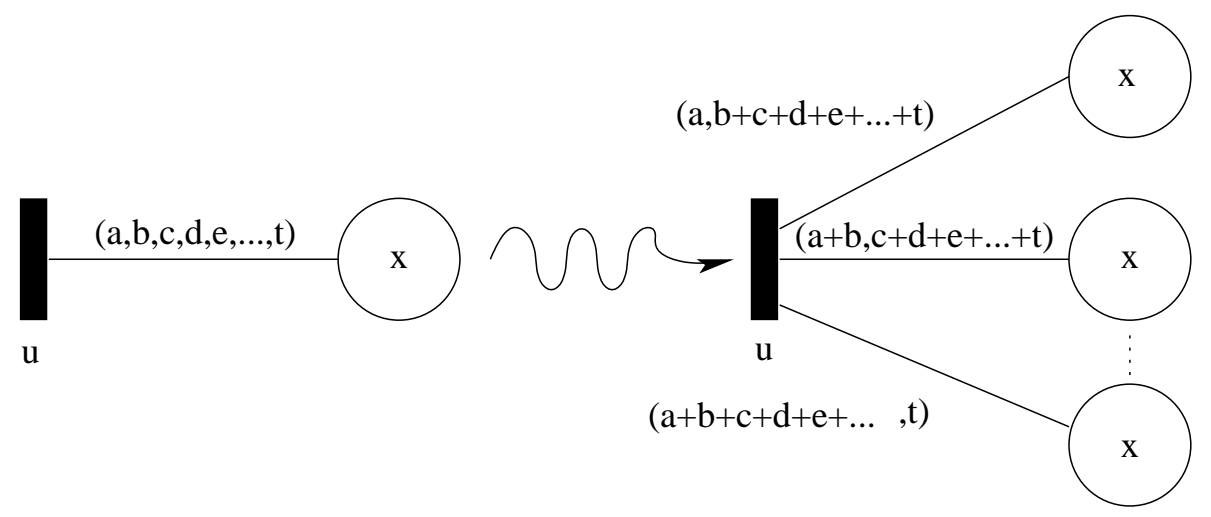

\section{Réseaux lexicographiques}

\subsection{Algèbres de Petri}

Jusqu'à présent les algèbres que nous avons choisies pour tenter d'étendre les réseaux de Petri présentent le défaut de perdre la notion de ressource. Un angle d'attaque beaucoup plus technique que l'on trouve dans (Badouel et al., 2005) consiste à caractériser les algèbres qui permettent de conserver la même règle de tir ainsi qu'un certain nombre de propriétés naturellement associées à la notion de ressource. En particulier, le monoïde $(\mathbb{N},+, 0)$ des entiers naturels qui est l'algèbre des places des réseaux de Petri doit en faire partie. Il nous faut donc un zéro, noté naturellement 0 , constante qui signifie l'absence de ressource et une relation d'ordre, notons la $\sqsubseteq$, pour pouvoir avoir une condition de franchissement similaire à celle des réseaux de Petri. Enfin nous devons être capable d'accumuler de la ressource, notons cette opération $\oplus$ que nous choisissons, à l'image de celle des réseaux de Petri, associative et commutative 
(il peut être intéressant d'ailleurs d'analyser le cas non commutatif), et de consommer ces mêmes ressources avec un opérateur $\ominus$ dit de résiduation, c'est-à-dire un pseudoinverse de $\oplus$ défini seulement lorsque l'on dispose de suffisamment de ressources (on ne fait $a \ominus b$ que lorsque $b \sqsubseteq a$ ). Supposons que nous disposions d'un réseau dans lequel les places et les flots prennent leurs valeurs dans une telle algèbre alors la relation de transition $M[t\rangle M^{\prime}$ peut s'écrire de manière tout à fait similaire à la relation de transition dans les réseaux de Petri usuels :

$M[t\rangle M^{\prime} \Leftrightarrow \forall p \in P, M(p) \sqsupseteq \operatorname{Pré}(p, t) \wedge M^{\prime}(p)=(M(p) \ominus \operatorname{Pré}(p, t)) \oplus \operatorname{Post}(p, t)$

Pour aller encore un peu plus loin dans l'analogie, on peut constater que le système de transitions associé à la relation de transition dans un réseau de Petri usuel est déterministe et co-déterministe, c'est-à-dire que la donnée de $M$ et de $t$ détermine $M^{\prime}$ et, inversement, la donnée de $M^{\prime}$ et de $t$ détermine $M$. En analysant les algèbres qui permettent de retrouver l'ensemble de ces caractéristiques, on constate qu'il est nécessaire qu'elles soient des monoïdes commutatifs de divisibilité $(a \sqsupseteq b \Longleftrightarrow \exists c$ tel que $a=b \oplus c)$, simplifiables $((a \oplus b) \ominus b=a)$ munies donc d'une opération de résiduation adjointe à l'addition (l'opérateur $\ominus$ ) et vérifiant $b \sqsubseteq a \Longrightarrow a=(a \ominus b) \oplus b$. On appellera de telles algèbres des algèbres de Petri commutatives. Le lecteur intéressé pourra trouver une démonstration complète du résultat qui suit dans (Badouel et al., 2007).

Théorème 4.1 Les algèbres de Petri commutatives cö̈ncident avec les cônes positifs des groupes commutatifs ordonnés en treillis.

\subsection{Définitions}

Les réseaux lexicographiques que nous définissons ci-après constituent un exemple de classe de réseaux fonctionnant sur une algèbre de Petri tout en constituant une extension stricte des réseaux de Petri. Ces réseaux acquièrent une importance toute particulière du fait de leur universalité dans le sens où tout réseau associé à une algèbre de Petri peut être simulé par un réseau lexicographique (Badouel et al., 2007).

Prenons maintenant le cône positif du produit lexicographique de $\mathbb{Z}$ avec luimême : $(\mathbb{Z} \circ \mathbb{Z})^{+}$. Le produit lexicographique (noté ici $\circ$ ) de $\mathbb{Z}$ avec lui-même est tout simplement le groupe produit $\mathbb{Z} \times \mathbb{Z}$ muni de l'ordre lexicographique :

$$
(u, v) \leq(z, t) \Longleftrightarrow u<v \text { ou }(u=z \text { et } v \leq t)
$$

Comme $\mathbb{Z}$ muni de son ordre habituel est une chaîne, $\mathbb{Z} \circ \mathbb{Z}$ muni de l'ordre lexicographique l'est aussi et est donc en particulier ordonné en treillis. Son cône positif est alors bien une algèbre de Petri commutative et

$$
(\mathbb{Z} \circ \mathbb{Z})^{+}=\{(x, y) \mid(x=0 \text { et } y \geq 0) \text { ou }(x>0 \text { et } y \in \mathbb{Z})\}
$$


Le produit lexicographique est associatif et on peut définir inductivement $\left(\mathbb{Z}^{n}\right)^{+}$ pour tout entier $n$ (on pourrait même l'étendre aux ordinaux) en posant $\mathbb{Z}^{n+1}=\mathbb{Z}^{n} \circ \mathbb{Z}$ et en le munissant de l'ordre lexicographique. Dans la suite nous appellons réseaux lexicographiques les réseaux fonctionnant sur l'algèbre $(\mathbb{Z} \circ \mathbb{Z})^{+}$.

Définition 4.1 (Réseau lexicographique) Un réseau lexicographique est un quintuplet $\left(P, T\right.$, Pré, Post, $\left.M_{0}\right)$ où $P$ est un ensemble fini de places, $T$ est un ensemble fini de transitions (disjoint de P), Pré et Post sont les relations de flot de $P \times T$ dans $(\mathbb{Z} \circ \mathbb{Z})^{+}$. On appellera marquage une application de $P$ dans $(\mathbb{Z} \circ \mathbb{Z})^{+}$et $M_{0}$ est le marquage initial.

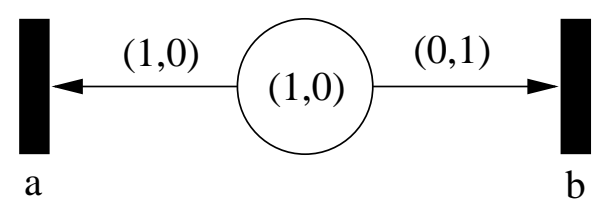

Figure 12. Réseau lexicographique

Comme pour les réseaux de Petri, le comportement d'un réseau lexicographique est complètement déterminé par la règle de tir.

Définition 4.2 (Règle de tir pour les réseaux lexicographiques) Soit $N=$ $\left(P, T\right.$, Pré, Post, $\left.M_{0}\right)$ un réseau lexicographique. Le tir de la transition $t$ à partir du marquage $M$ est autorisé si $\forall p \in P, M(p) \geq \operatorname{Pré}(p, t)$. Dans ce cas on peut franchir $t$ ce qui donne le nouveau marquage $M^{\prime}$ défini par $\forall p \in P, M^{\prime}(p)=M(p)-\operatorname{Pré}(p, t)+\operatorname{Post}(p, t)$ (où - est l'opération de résiduation associée naturellement $\grave{a}+)$.

Nous reprenons par ailleurs les définitions usuelles sur les réseaux de Petri que nous étendons directement aux réseaux lexicographiques : séquence de tirs, langage d'un réseau $N$ que nous noterons $L(N)$, ensemble d'accessibilité noté $R(N)$, graphe des marquages noté $M G(N) \ldots$

Par exemple, le langage du réseau de la figure 12 est l'ensemble des préfixes de $a+b^{*}$ qui ne peut-être le langage d'un réseau de Petri. En effet, supposons que l'on dispose d'un réseau de Petri ayant un tel langage. $b$ étant franchissable indéfiniment à partir du marquage initial, dans chaque place, b produit plus de ressources qu'elle n'en consomme et, par conséquent, par monotonie, $a$ devrait être a fortiori franchissable après l'exécution de $b$. Ceci nous conduit à la proposition suivante.

Proposition 4.1 Les réseaux lexicographiques sont une extension stricte des RdP. 
En fait, on peut même exhiber une famille infinie de réseaux strictement emboîtés : les réseaux lexicographiques sur les algèbres $\left(\mathbb{Z}^{n}\right)^{+}$. Pour s'en convaincre contentonsnous d'exhiber le réseau sur $(\mathbb{Z} \circ \mathbb{Z} \circ \mathbb{Z})^{+}$de la figure 13 , non modélisable par un réseau lexicographique sur $(\mathbb{Z} \circ \mathbb{Z})^{+}$. Schématiquement, $a, b$ et $c$ consomment tous trois strictement de la ressource, $b$ dans une proportion infiniment négligeable par rapport à $a$ ainsi que $c$ par rapport à $b$. Dans un réseau lexicographique seuls deux ordres de grandeur de consommation sont modélisables.

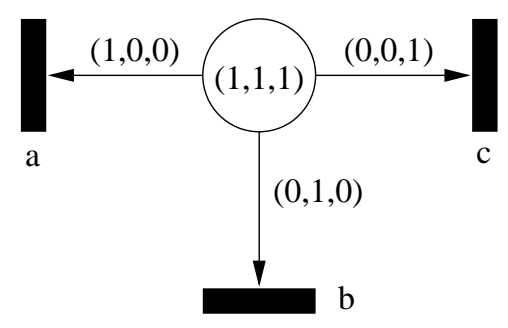

Figure 13. Réseau sur $(\mathbb{Z} \circ \mathbb{Z} \circ \mathbb{Z})^{+}$non modélisable par un réseau sur $(\mathbb{Z} \circ \mathbb{Z})^{+}$

\subsection{Réseaux lexicographiques et machines de Turing}

Un outil fondamental qui permet de résoudre nombre de problèmes de décision pour les réseaux de Petri est l'arbre de couverture défini par (Karp et al., 1969). Cependant le caractère fini de cette construction est lié au fait que l'ordre naturel sur $\mathbb{N}^{|P|}$ est bien fondé, ce qui n'est plus le cas pour $(\mathbb{Z} \circ \mathbb{Z})^{+}$. A priori, définir un arbre de couverture pour les réseaux lexicographiques semble difficile. On peut alors se demander si les réseaux lexicographiques ont la puissance des machines de Turing, auquel cas tous les problèmes de décidabilité non triviaux (terminaison, caractère borné, accessibilité...) deviennent indécidables. Une classe de réseau qui a cette puissance est celle des réseaux de Petri avec arcs inhibiteurs. Comparons-les aux réseaux lexicographiques. Commençons par remarquer que certains réseaux lexicographiques n'ont pas un comportement de réseau de Petri à arcs inhibiteurs. En effet, prenons le réseau de la figure 14 : son langage est l'ensemble des préfixes de $a b+b^{*}$ et ne peut être le langage d'un réseau de Petri avec arcs inhibiteurs. En effet, comme pour les réseaux de Petri standard, le fait que $b$ soit indéfiniment tirable à partir du marquage initial impose à $b$ de faire croître les marquages, c'est-à-dire que $M[b\rangle M^{\prime} \Longrightarrow M \leq M^{\prime}$, la présence possible d'arcs inhibiteurs entre certaines places et la transition $b$ imposant simplement que le marquage de ces places reste à zéro (donc pas d'arcs Post correspondants). Cependant après $a, b$ n'est tirable qu'une fois et comme $b$ à une action croissante sur les marquages, c'est qu'au moins une place est en self-loop avec la transition $b$ via un arc inhibiteur et un arc Post valué par une valeur strictement positive. Mais alors $b$ ne serait plus tirable indéfiniment à partir du marquage initial... 


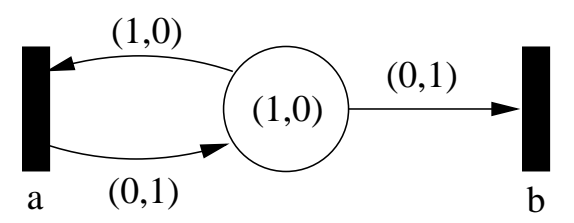

Figure 14. Réseau lexicographique sans RdP à arcs inhibiteurs équivalent

Théorème 4.2 Les réseaux lexicographiques contiennent strictement les réseaux de Petri à arcs inhibiteurs.

Avant de passer à la preuve, fixons la terminologie en rappelant la définition d'un réseau de Petri à arcs inhibiteurs.

Définition 4.3 (Réseau de Petri à arcs inhibiteurs) Un réseau de Petri à arcs inhibiteurs est un quadruplet $N=(P, T$, Pré, Post) où P, T et Post sont définis de manière identique à celle des réseaux de Petri, mais Pré est une application de $P \times T$ dans $\mathbb{N} \cup\{\emptyset\}$ où $\emptyset$ est un symbole. Une transition $t$ est franchissable pour un marquage $M \in \mathbb{N}^{P}$ lorsque pour toute place $p, M(p) \geq \operatorname{Pré}(p, t)$ si Pré $(p, t) \in \mathbb{N}$ ou $M(p)=0$ si Pré $(p, t)=\emptyset$. Si t est franchissable à partir de $M$ alors le marquage $M^{\prime}$ obtenu est défini par :

$$
\forall p \in P, M^{\prime}(p)= \begin{cases}M(p)-\operatorname{Pré}(p, t)+\operatorname{Post}(p, t) & \text { si } \operatorname{Pré}(p, t) \in \mathbb{N} \\ M(p)+\operatorname{Post}(p, t) & \text { si } \operatorname{Pré}(p, t)=\emptyset\end{cases}
$$

Preuve Soit $N=\left(P, T\right.$, Pré, Post, $\left.M_{0}\right)$ un réseau de Petri marqué à arcs inhibiteurs. Commençons par distinguer les éléments de $P$. On pose $P_{1}=\{p \in P \mid \forall t \in$ $T, \operatorname{Pré}(p, t) \in \mathbb{N}\}$ et $P_{2}$ son complémentaire dans $P$. $P_{2}$ contient donc les places qui sont reliées à au moins une transition par un arc inhibiteur.

Soit maintenant $N^{\prime}=\left(P^{\prime}, T\right.$, Pré $^{\prime}$, Post $\left.^{\prime}, M_{0}^{\prime}\right)$ le réseau lexicographique défini ainsi : $P^{\prime}=P \cup P_{2}^{\prime}$ avec $P_{2}^{\prime}$ un ensemble disjoint de $P$ tel que $\left|P_{2}^{\prime}\right|=\left|P_{2}\right|$. Pour des raisons pratiques on notera $p^{\prime}$, pour chaque place $p$ de $P_{2}$, les places de $P_{2}^{\prime}$, et on dira que $p^{\prime}$ est la place négative associée à $p$. Par ailleurs :

$\forall p \in P, M_{0}^{\prime}(p)=\left(0, M_{0}(p)\right)$

$\forall p^{\prime} \in P_{2}^{\prime}, M_{0}^{\prime}\left(p^{\prime}\right)=\left(1,-M_{0}(p)\right)$

c'est-à-dire qu'une place négative a pour marquage 1 suivi du négatif (l'inverse) du marquage de la place du réseau de Petri initial alors que tout autre place contient 0 suivi du marquage initial de la place en question.

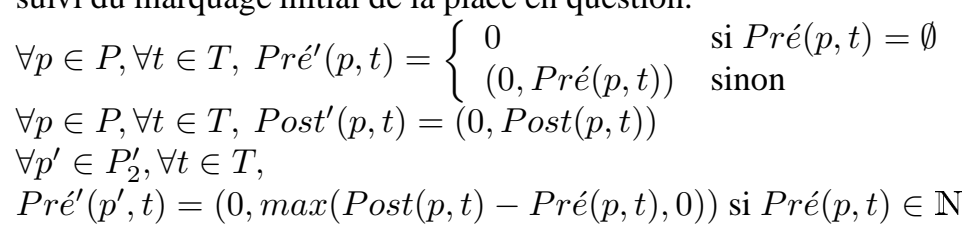


$\operatorname{Pré}^{\prime}\left(p^{\prime}, t\right)=(1,0)$ si $\operatorname{Pré}(p, t)=\emptyset$

$\operatorname{Post}^{\prime}\left(p^{\prime}, t\right)=(0, \max (\operatorname{Pré}(p, t)-\operatorname{Post}(p, t), 0))$ si $\operatorname{Pré}(p, t) \in \mathbb{N}$

$\operatorname{Post}^{\prime}\left(p^{\prime}, t\right)=(1,-\operatorname{Post}(p, t))$ si Pré $(p, t)=\emptyset$

En fait, les transitions agissent de la même manière que dans le réseau de Petri de départ mais uniquement sur la deuxième composante des marquages des places $p \in P$ et de manière inverse sur les places négatives associées. Enfin l'arc inhibiteur et l'arc Post associé s'il existe sont remplacés par une self-loop.

La figure 15 donne un exemple de réseau lexicographique équivalent à un réseau de Petri à arcs inhibiteurs obtenu par l'algorithme qui vient d'être exposé.

La correspondance $\sigma$ entre les marquages de $N$ et de $N^{\prime}$ est définie par l'application qui envoie tout marquage $M$ de $N$ sur le marquage $M^{\prime}$ de $N^{\prime}$ tel que

$$
\begin{gathered}
\forall p \in P, M^{\prime}(p)=(0, M(p)) \\
\forall p^{\prime} \in P_{2}^{\prime}, M^{\prime}\left(p^{\prime}\right)=(1,-M(p))
\end{gathered}
$$

On constate que $M^{\prime}$ est bien un élément de $(\mathbb{Z} \circ \mathbb{Z})^{+}$car $M \in \mathbb{N}^{P}$.

On se convainc aisément ensuite que cette correspondance est préservée par tir de transitions. Enfin, l'inclusion stricte est donnée par le réseau de la figure 14. $\diamond$

Il est intéressant de constater que le test à zéro dans le réseau de Petri à arcs inhibiteurs initial est également remplacé par un test à zéro dans le réseau lexicographique associé. En effet une place $p$, de marquage $k$, reliée par un arc inhibiteur à une transition $a$ est remplacée par deux places $p$ et $p^{\prime}$. Dans le réseau lexicographique $a$ ne sera tirable que si $M(p)=(1,-k) \geq(1,0)$, mais comme $M\left(p^{\prime}\right)=(0, k)$, a sera tirable si et seulement si $M\left(p^{\prime}\right)=0$ (ou encore si et seulement si $M(p)=(1,0)$ ).

Du théorème précédent découle le corollaire suivant.

Corollaire 4.1 Les problèmes de la terminaison, de la couverture, du caractère borné d'un réseau, du caractère borné d'une place, du caractère structurellement borné, de l'accessibilité, du blocage, de la vivacité et de la t-vivacité sont indécidables.

\subsection{Réseaux lexicographiques bornés}

Bien que le caractère borné d'un réseau soit indécidable, on peut se demander ce que sont les réseaux lexicographiques bornés. Pour lever toute ambiguïté, notons que, contrairement aux réseaux de Petri et afin de préserver la même notion, le caractère borné d'un réseau lexicographique ne peut être lié à l'ordre de l'algèbre sous-jacente, car l'ordre lexicographique n'est pas bien fondé.

Définition 4.4 (place bornée) Soit $N=\left(P, T\right.$, Pré, Post, $\left.M_{0}\right)$ un réseau lexicographique. Une place $p \in P$ est dite bornée si $\{M(p) / M \in R(N)\}$ est fini.

Définition 4.5 (réseau lexicographique borné) Soit $N=\left(P, T\right.$, Pré, Post, $\left.M_{0}\right)$ un réseau lexicographique. $N$ est borné si toute place $p \in P$ est bornée. 


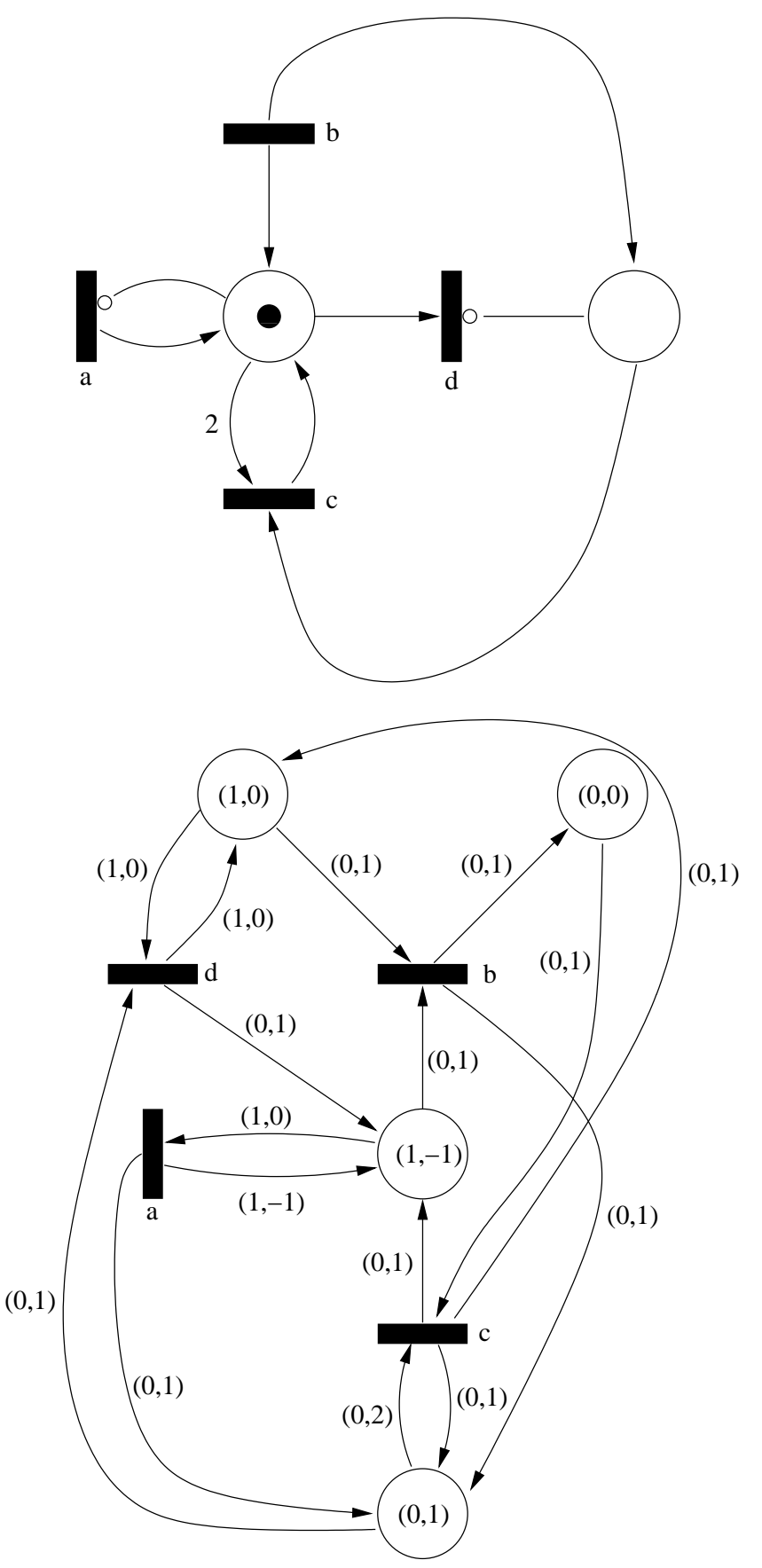

Figure 15. RdP à arcs inhibiteurs et son réseau lexicographique équivalent 
Si $x \in \mathbb{Z} \times \mathbb{Z}$, on notera $x(1)$ sa première composante et $x(2)$ sa deuxième.

Théorème 4.3 (Caractérisation des réseaux lexicographiques bornés) Les réseaux lexicographiques bornés sont les réseaux de Petri bornés.

Preuve Tout réseau de Petri étant un réseau lexicographique, le sens réciproque est trivial. Soit maintenant $N=\left(P, T\right.$, Pré, Post, $\left.M_{0}\right)$ un réseau lexicographique borné. Par conséquent $R(N)$ l'ensemble des marquages accessibles de $N$ est fini. Soit $N^{\prime}=$ $\left(P, T\right.$, Pré $^{\prime}$, Post $\left.^{\prime}, M_{0}^{\prime}\right)$ le réseau de Petri dont la construction est détaillée ci-dessous.

Pour chaque place $p$ de $N$ on ordonne l'ensemble constitué des $M(p)$ pour $M \in$ $R(N)$, des Pré $(p, t)$ pour $t \in T$ et des $\operatorname{Post}(p, t)$ pour $t \in T$. Ceci nous donne pour chaque $p \in P$ une liste finie triée de manière strictement croissante que l'on notera $L_{p}$, on notera sa longueur $n_{p}$ et $\left(\left(a_{i, p}, b_{i, p}\right)\right)_{i \in\left\{1,2, \ldots, n_{p}\right\}}$ ses éléments. L'idée est de chercher pour chaque place $p$ de $P$ un $\alpha_{p} \in \mathbb{Z}$ tel que la suite finie $\left(a_{i, p} \alpha_{p}+\right.$ $\left.b_{i, p}\right)_{i \in\left\{1,2, \ldots, n_{p}\right\}}$ soit strictement croissante et dans $\mathbb{N}$. Par conséquent il est nécessaire que d'une part $a_{1, p} \alpha_{p}+b_{1, p} \geq 0$ c'est-à-dire que $\alpha_{p} \geq-\frac{b_{1, p}}{a_{1, p}}$ si $a_{1, p}$ est non nul, et d'autre part, que pour tout $i$ entre 1 et $n_{p}-1, a_{i, p} \alpha+b_{i, p}<a_{i+1, p} \alpha+b_{i+1, p}$. Cette dernière inégalité étant vérifiée si $a_{i, p}=a_{i+1, p}$, on ne s'occupe que des cas pour lesquels $a_{i, p}<a_{i+1, p}$. Par conséquent il est nécessaire que $\alpha_{p}>\frac{b_{i, p}-b_{i+1, p}}{a_{i+1, p}-a_{i, p}}$ pour tout $i$ entre 1 et $n_{p}-1$ tel que $a_{i, p}<a_{i+1, p}$. Il suffit donc de choisir $\alpha_{p}$ tel que

$$
\alpha_{p}=\left\{\begin{array}{c}
\max \left(\left\{\left\lfloor\frac{b_{i, p}-b_{i+1, p}}{a_{i+1, p}-a_{i, p}}\right\rfloor+1 \mid a_{i, p}<a_{i+1, p} \text { et } 1 \leq i \leq n_{p}-1\right\}\right. \\
\left.\cup\left\{\left\lceil-\frac{b_{1, p}}{a_{1, p}}\right\rceil \mid a_{1, p}>0\right\}\right) \\
1 \text { si l'ensemble ci-dessus est vide }
\end{array}\right.
$$

Le second cas n'intervenant que si $a_{i, p}=0$ pour tout $i$ dans $\left\{1, \ldots, n_{p}\right\}$.

On peut maintenant définir $M_{0}^{\prime}$ :

$$
\forall p \in P, M_{0}^{\prime}(p)=M_{0}(p)(1) \alpha_{p}+M_{0}(p)(2)
$$

ainsi que Pré ${ }^{\prime}$ et Post $^{\prime}$ :

$$
\begin{gathered}
\forall p \in P, \forall t \in T, \operatorname{Pré}^{\prime}(p, t)=\operatorname{Pré}(p, t)(1) \alpha_{p}+\operatorname{Pré}(p, t)(2) \\
\forall p \in P, \forall t \in T, \operatorname{Post}^{\prime}(p, t)=\operatorname{Post}(p, t)(1) \alpha_{p}+\operatorname{Post}(p, t)(2)
\end{gathered}
$$

La correspondance entre les marquages est donnée par l'application $\sigma$ de $R(N)$ dans $R\left(N^{\prime}\right)$ qui à tout marquage $M$ de $N$ associe le marquage $M^{\prime}$ de $N^{\prime}$ tel que $\forall p \in P, M^{\prime}(p)=M(p)(1) \alpha_{p}+M(p)(2)$. On conclut en montrant que cette correspondance est préservée par tir de transition. $\diamond$ 
Appliquons l'algorithme que nous venons de voir au réseau suivant :

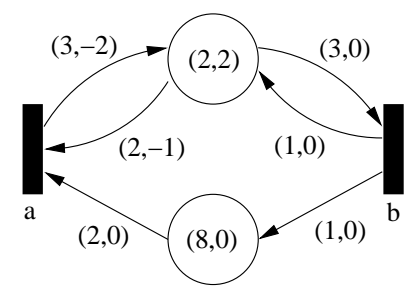

$L_{a}=\{(1,0),(1,1),(2,-2),(2,0),(2,2),(3,-2),(3,-1),(3,0),(3,1),(4,-2)$, $(4,0),(5,-1),(6,-2)\}, \alpha_{a}=5, L_{b}=\{(0,0),(1,0),(2,0),(3,0),(4,0),(5,0)$, $(6,0),(7,0),(8,0)\}$ et $\alpha_{b}=1$. Par conséquent, le réseau de Petri équivalent est :

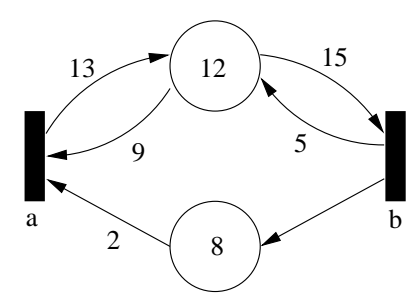

Notons que la construction précédente est aisément généralisable aux réseaux sur l'algèbre $\left(\mathbb{Z}^{n}\right)^{+}$pour $n \geq 1$. En effet, soit $L=\left(l_{i}\right)_{1 \leq i \leq k}$ une liste finie d'éléments distincts de $\left(\mathbb{Z}^{n}\right)^{+}$triés par ordre croissant. Il est alors facile de trouver un $\alpha \in \mathbb{Z}$ (par essais successifs de valeurs de plus en plus grandes en partant de la valeur 0 par exemple) tel que la suite finie $\left(\left(l_{i}(1) \alpha^{n-1}+l_{i}(2) \alpha^{n-2}+\ldots+l_{i}(n-1) \alpha+\right.\right.$ $\left.\left.\left.l_{i}(n)\right)\right)\right)_{1 \leq i \leq k}$ soit strictement croissante dans $\mathbb{N}$ car on ramène le problème à un système d'inéquations de la forme $P(\alpha)>0$ ou (pour une d'entre elles) $P(\alpha) \geq 0$ avec $P$ polynôme en $\alpha$ dont le coefficient de poids fort est strictement positif. Par conséquent, les $P$ divergent vers $+\infty$ en $+\infty$, donc on peut trouver un $\alpha$ pour chaque polynôme vérifiant l'inégalité associée. La valeur maximale de ces $\alpha$ convient alors.

Corollaire 4.2 Les réseaux lexicographiques bornés sur $\left(\mathbb{Z}^{n}\right)^{+}(n \geq 1)$ sont les réseaux de Petri bornés.

\section{Conclusion et perspectives}

Dans cet article nous proposons dans un premier temps de changer l'algèbre des places des réseaux de Petri par un groupe privé de l'élément neutre. Les strict-groupnets ainsi obtenus permettent de généraliser les réseaux de Petri purs en choisissant comme groupe l'ensemble des entiers relatifs $\mathbb{Z}$ et permettent également de retrouver les réseaux élémentaires. L'adjonction d'un arc dit « inconditionnel » conduit aux 
group-nets. Ces réseaux étendent les réseaux de Petri $n$-bornés, les réseaux de Petri places/transitions usuels ainsi que les strict-group-nets. Nous montrons que les problèmes de savoir si un réseau est borné et si une place d'un réseau est bornée sont décidables à travers une notion d'arbre de couverture. Nous donnons également une présentation unifiée des $\mathbb{Z}$-nets et des strict- $\mathbb{Z}$-nets à travers les $\mathbb{Z}_{2}$-nets. De nombreuses questions restent encore ouvertes sur ces réseaux. Par exemple : peut-on décider de leur régularité (au sens de la théorie des langages), de leur accessibilité ? Y a t-il des liens entre groupe-produit et produit de réseaux ? Nous savons que les réseaux de Petri ont des liens étroits avec les groupes sans torsion, peut-on exploiter ce fait avec les group-nets?

Une deuxième partie est consacrée aux réseaux lexicographiques issus d'une approche basée sur une axiomatisation de la notion de ressource et de la règle de tir. Ces réseaux constituent une extension stricte des réseaux de Petri mais ont malheureusement la puissance des machines de Turing rendant indécidable tout problème non trivial. Cependant, pour parachever l'étude de ces réseaux, nous montrons que les réseaux lexicographiques bornés sont les réseaux de Petri bornés. Par ailleurs la notion de ressource qu'on y trouve est étrange : il est possible de consommer strictement et indéfiniment! Si les réseaux lexicographiques en tant que modèle semblent bien cernés, il demeure de nombreuses perspectives de développement vis à vis des algèbres de Petri. On peut se pencher sur le cas des algèbres de Petri non-commutatives pour distinguer des réseaux « à files » ou « à piles » (Badouel et al., 2003), d'algèbres de Petri non régulières où l'algèbre des places et les valeurs des relations de flot n'opèrent pas sur la même algèbre et sont connectées par une relation de satisfaction ou encore d'algèbres « enrichies » permettant de mesurer le degré de franchissabilité d'une transition.

\section{Bibliographie}

André C., Systèmes à évolutions parallèles : modélisation pour réseaux de Petri à capacité et analyse par abstraction, Université de Nice. Thèse d'état, 1981.

Araki T., Kasami T., « Some decision problems related to the reachability problem for Petri nets », Theoretical Computer Science, vol. 3, n 1, p. 85-104, 1977.

Badouel E., Chenou J., « Nets Enriched over Closed Monoidal Structures », Lecture Notes in Computer Science, vol. 2679, ICATPN'03, p. 64-81, 2003.

Badouel E., Chenou J., Guillou G., « Petri Algebras », Lecture Notes in Computer Science, vol. 3580, ICALP'2005, p. 742-754, 2005.

Badouel E., Chenou J., Guillou G., « An axiomatization of the token game based on Petri Algebras », Fundamenta Informaticae, vol. 77, n 3, p. 187-215, 2007.

Badouel E., Darondeau P., « Theory of regions », Lecture Notes in Computer Science, vol. 1491, p. 529-586, 1999.

David R., Alla H., « Continuous and Hybrid Petri Nets », Journal of Circuits Systems Computers, vol. 8, n 1, p. 159-188, 1998. 
Dufourd C., Finkel A., Schnoebelen P., « Reset Nets Between Decidability and Undecidability », Lecture Notes in Computer Science, vol. 1443, ICALP'98, p. 103-115, 1998.

Genrich H., Lautenbach K., Thiagarajan P., « Elements of General Net Theory », Lectures Notes in Computer Science, vol. 84, Advanced Course on General Net Theory of Processes and Systems : Net Theory and Applications, p. 21-163, 1979.

Guillou G., Synthèse des $\mathbb{Z} / 2 \mathbb{Z}$-nets, Technical report, EA2215, 2003.

Guillou G., « Group-nets and strict-group-nets », Petri Net Newsletter, vol. 67, p. 17-25, 2004.

Hack M., Petri Net Languages, CSG Memo 124, Project MAC, MIT, 1975.

Haddad S., Poitrenaud D., « Recursive Petri Nets - Theory and Application to Discrete Event Systems », Acta Informatica, vol. 44, n 7-8, p. 463-508, 2007.

Hopcroft J., Pansiot J., « On the reachability problem for 5-dimensional vector addition systems », Theoretical Computer Science, vol. 8, p. 135-159, 1979.

Jensen K., Coloured Petri nets. Basic Concepts, Analysis Methods and Practical Use, vol. 1 of EATCS Monographs on Theoritical Computer Science, Springer Verlag, 1992.

Juhás G., Algebraically Generalised Petri Nets, PhD thesis, Institute of Control Theory and Robotics. Slovak Academy of Sciences, 1998.

Karp R., Miller R., « Parallel Program Schemata », Journal of Computer and System Sciences, vol. 3, n² 2, p. 147-195, 1969.

Lazić R., Newcomb T., Ouaknine J., Roscoe A., Worell J., « Nets with Tokens Which Carry Data », Lecture Notes in Computer Science, vol. 4546, ICATPN'07, p. 301-320, 2007.

Mayr R., « Process Rewrite Systems », Information and Computation, vol. 156, n 1-2, p. $264-$ 286, 2000.

Meseguer J., Montanari U., « Petri nets are monoids », Information and Computation, vol. 88, $\mathrm{n}^{\circ} 2$, p. 105-155, 1990.

Moalla M., Pulou J., Sifakis J., « Réseaux de Petri synchronisés », Revue RAIRO Automatique, vol. 12, n 2, p. 103-130, 1978.

Murata T., « Petri nets : Properties, Analysis and Applications », Proceedings of the IEEE, vol. $77, n^{\circ} 4$, p. 541-588, 1989.

Padberg J., Abstract Petri Nets : Uniform Approach and Rule-Based Refinement, PhD thesis, Technical University of Berlin, 1996.

Schmitt V., « Flip-flop Nets », Lectures Notes in Computer Science, vol. 1046, STACS'96, p. 517-528, 1996.

Valk R., « Self-modifying nets, a natural extension of Petri nets », Lecture Notes in Computer Science, vol. 62, ICALP'78, p. 464-476, 1978. 